\title{
A Measure-Theoretic Model for Collective Cell Migration and Aggregation
}

\author{
A. Colombi, M. Scianna, L. Preziosi * \\ Department of Mathematics, Politecnico di Torino, Corso Duca degli Abruzzi 24, 10129 Torino, Italy
}

\begin{abstract}
The aim of this paper is to present a measure-theoretic approach able to derive an Eulerian model of the dynamics of a cell population with a finite number of cells out of a microscopic Lagrangian description of the underlying cellular particle system. By looking at the spatial distribution of cells in terms of a time-evolving probability measure, rather than at individual cell paths, an ensemble representation of the cell colony is obtained, which can then result either in discrete, continuous, or hybrid approaches according to the spatial structure of such a probability measure. Remarkably, such an approach does not call for any assumption on the number of cells taken into account, thus providing consistency of the same modeling framework across all levels of representation. In addition, it is suitable to cope with the often ambiguous translation of microscopic arguments (i.e., cell dimensions and interaction radii) into macroscopic descriptions. The proposed approach, also extended to the case of multiple coexisting cell populations, is then tested with sample simulations that provide a useful sensitivity analysis of the model parameters.
\end{abstract}

Keywords and phrases: cell populations, probability measures, discrete vs. continuous descriptions

Mathematics Subject Classification: 35Q70, 35Q92, 92C17

\section{Introduction}

The evolution of a living system is in general a complex and multiscale phenomenon involving many different but interconnected processes, that occur at different spatio-temporal levels. In particular, macroscopic dynamics of large cell aggregates and tissues results from microscopic behavior of single component cells, which are able to sense and actively interact with the surrounding environment in a non strictly mechanical manner. This gives rise to entirely different dynamics from those seen in standard systems of inert matter (e.g., fluids, gases), whose particles respond passively to relatively simple physical rules. In this respect, the evolution of a cellular ensemble is not the simple superposition of individual behaviors. In fact, complex interactions arise among cells, leading to the spontaneous emergence of collective patterns. The study of such an intricate temporal and spatial flow of information highlights the need for experimental researchers to work in multiscale environments, that are capable of ensemble representations of biological systems while still linking their dynamics to the actual cell-based behavior.

*Corresponding author. E-mail: luigi.preziosi@polito.it

(C) EDP Sciences, 2015 
With respect to the existing multiscale frameworks proposed in the literature to reproduce the evolution of cell populations (see, for instance, $[2,17,19,21,26,36,37,39]$ ), we here propose an approach based on measure-theoretic probabilistic tools, recently developed and successfully applied to crowd dynamics $[4,14-16,35]$. The leading idea is that the underlying physical system (i.e., the cell aggregate) is always regarded as a granular one, as we admit that the positions of the single individuals are defined by random variables. The spatio-temporal evolution of the overall cell population is instead described by a probability measure (law) attached to each of these variables rather than by the variables themselves. In this way, it is possible to naturally switch from a Lagrangian-phenomenological viewpoint to an Eulerian description without any further assumptions on the cell number (or on the cell dimensions), which is a crucial novelty of the proposed approach with respect to most methods presented in literature.

Then, another key feature is that our theoretical framework is expected to provide consistent mathematical tools to address the multiscale issues posed by biomedical/biological problems. In fact, the description of the system in terms of an abstract measure does not force an a priori choice of the scale of representation, which can be a posteriori deferred only to the specific structure given to the probability distribution (i.e., atomic, absolutely continuous with respect to Lebesgue, or even hybrid). This is of particular relevance also in the case of a system composed by multiple coexisting populations, each of them requiring (within the same simulation environment) a specific level of description according to its biological properties.

After the above general presentation, the contents of the paper can now be outlined more precisely. This Introduction is concluded by a useful collection of the main notations and theoretical notions used in the rest of the paper. In Section 2, the mathematical structures based on time-evolving probability distributions are introduced for modeling cell population dynamics by the measure-theoretic approach. In particular, we first introduce a single cell phenomenological model and then devise the proper evolution equation for the measure of the overall aggregate. The measure-theoretic general framework is further exploited to obtain microscopic/discrete, macroscopic/continuous as well as hybrid descriptions of the system. In Section 3, a computational analysis of our method is performed by means of illustrative examples, which study the influence on the resulting cell dynamics of both the model parameters and the scale of representation used. The subsequent Sections 4-5 deal with the model extension to multiple and coexisting cell populations. In particular, we focus on the case of a small group of activated/differentiated cells, suitably described with a discrete viewpoint, which behave as guidance leaders for a mass of undifferentiated individuals, approached with a continuum approximation. Finally, conclusions and possible developments of the method are drawn in Section 6.

\section{Mathematical model}

Notations and background. We use the space $\mathbf{x} \in \mathbb{R}^{n}$ and the time $t \in[0, T], T>0$ being a certain final time (possibly $+\infty$ ), as independent variables. The space dimension is $n \leq 3$ for physical reasons.

A function $f$ of $t, \mathbf{x}$ is generically defined on $[0, T] \times \mathbb{R}^{n}$. Instead of the classical $f(t, \mathbf{x})$ we prefer the notation $f_{t}(\mathbf{x})$, which means that we are looking at $f$ as parameterized by time (thus $f_{t}$, for fixed $t$, is a function defined in $\left.\mathbb{R}^{n}\right)$. In particular, the subscript $t$ will never stand for time derivative.

The indicator function of a set $A$ is $1_{A}$, thus $1_{A}(\mathbf{x})=1$ if $\mathbf{x} \in A, 1_{A}(\mathbf{x})=0$ if $\mathbf{x} \notin A$.

We endow $\mathbb{R}^{n}$ with the Borel $\sigma$-algebra $\mathcal{B}\left(\mathbb{R}^{n}\right)$. We work with probability measures defined on $\mathcal{B}\left(\mathbb{R}^{n}\right)$ and parameterized by time $t$, that we denote by $\mu_{t}$ (e.g., the subscript $t$ does not stand for time derivative). More precisely, if $\mathcal{P}\left(\mathbb{R}^{n}\right)$ is the space of probability measures on $\mathbb{R}^{n}$ then $\mu_{t}$ is a mapping $t \mapsto \mathcal{P}\left(\mathbb{R}^{n}\right)$. Thus $\mu_{t}$, for fixed $t$, is defined in $\mathbb{R}^{n}$.

The integral on $\mathbb{R}^{n}$ of a function $f$ with respect to $\mu_{t}$ is denoted by $\int_{\mathbb{R}^{n}} f d \mu_{t}$ or, if it is necessary to specify the variable of integration, by $\int_{\mathbb{R}^{n}} f(\mathbf{x}) d \mu_{t}(\mathbf{x})$.

The leading idea of this approach is that models of biological systems can be suitably designed at the physically natural and intuitive level of the single component cells. This description can then be translated to the more abstract level of a time-evolving measure representing the distribution in space of the mass of the overall cell aggregate. In other words, the task is to devise a proper evolution model for such a global measure starting with the law attached to the single individuals. From such a perspective, 
the underlying physical system is always regarded as a granular one, thereby allowing to directly and coherently refer experimental quantities and parameters to the cellular scale.

In our approach, the evolution of a single cell is described at the suitable individual scale by means of a variable $\mathbf{X}_{t}, t \geq 0$, which takes values in the measurable space $\left(\mathbb{R}^{n}, \mathcal{B}\left(\mathbb{R}^{n}\right)\right)$, see [44].

The mass of the overall cellular aggregate is instead represented mesoscopically by a Radon positive measure $\mu_{t}$, that we assume to be defined on the Borel $\sigma$-algebra $\mathcal{B}\left(\mathbb{R}^{n}\right)$. For any measurable set $E \in$ $\mathcal{B}\left(\mathbb{R}^{n}\right)$, the value $\mu_{t}(E) \geq 0$ measures therefore the mass of cells located in $E$ at time $t \geq 0$. In particular, the $\sigma$-additivity of $\mu_{t}$ directly translates the concept of the additivity of physical masses. In our approach, we assume, in a first approximation, that cells can not undergo mitosis or apoptosis, so that the total mass of the system is preserved in time, i.e.,

$$
\mu_{t}\left(\mathbb{R}^{n}\right)=N \quad \forall t \geq 0
$$

where $N$ is the constant finite number of cells.

Summing up, $\mathbf{X}_{t}$ is characteristic of an individual-phenomenological viewpoint, whereas the measure $\mu_{t}$ of a collective-mathematical description.

\subsection{Single cell phenomenological model}

The position in space of a generic component cell, hereafter called test cell, is described by the random variable $\mathbf{X}_{t}, t \geq 0$. If an initial position $\mathbf{X}_{0}=\mathbf{x}_{0} \in \mathbb{R}^{n}$ is assigned, the mapping $t \mapsto \mathbf{X}_{t}\left(\mathbf{x}_{0}\right)$ represents therefore the trajectory of the cell placed in $\mathbf{x}_{0}$ at the initial time $t=0$, i.e., we are considering a Lagrangian point of view. A practical assumption for reducing the complexity of the problem is that the cells within the population are indistinguishable from one another, in the sense that if initially two cells switch their position, the dynamics observed at future times is the same.

To approach the dynamics of the test cell, we recall that biological individuals are not passively prone to Newtonian laws of inertia, but are able to actively develop behavioral strategies which depend both on specific internal stimuli and on the interaction with the external environment (other cells, chemical fields or extracellular matrix components). Furthermore, in extremely viscous regimes, such as the biological environments themselves, the velocity of moving individuals and not their acceleration is typically proportional to the sensed forces (overdamped force-velocity response, characteristic of several Individual-Based Models $[20,22,37])$.

These concepts, also developed for other systems of living entities, such as human crowds or swarms $[15,16]$, can be translated into mathematical terms by a direct modeling of the velocity of the cells. In particular, we here assume that the dynamics of a cell in an aggregate result from i) a specific directional movement and ii) its interactions with the surrounding individuals. A prototype for the evolution equation of the variable $\mathbf{X}_{t}$ then writes as:

$$
\dot{\mathbf{X}}_{t}=\mathbf{v}_{d i r}\left(\mathbf{X}_{t}\right)+\mathbf{v}_{i n t}\left(\mathbf{X}_{t}\right)=\mathbf{v}_{d i r}\left(\mathbf{X}_{t}\right)+\int_{\mathbb{R}^{n} \backslash\left\{\mathbf{X}_{t}\right\}} \mathbf{K}\left(\mathbf{X}_{t}, \mathbf{y}\right) d \mu_{t}(\mathbf{y})
$$

where $\mathbf{v}_{\text {dir }}$ denotes a cell preferred velocity and $\mathbf{v}_{\text {int }}$ an interaction-dependent velocity (that will be described in more details below).

Cell preferred velocity. The term $\mathbf{v}_{\text {dir }}: \mathbb{R}^{n} \mapsto \mathbb{R}^{n}$ is a local preferred velocity, given for each individual. It implements cell preference to follow one direction with respect to the others, which may arise from intercellular stimuli and/or from the presence of specific environmental factors. In particular, such extracellular guidance cues give rise to migratory phenomena generally called taxis, which include:

- chemotaxis, where the preferred motion field is established by the spatial distribution of some diffusive chemical the cell is sensitive to, i.e.,

$$
\mathbf{v}_{d i r}\left(\mathbf{X}_{t}\right)= \pm \alpha_{c}\left(\mathbf{X}_{t}\right) \frac{\left|\nabla c_{t}\left(\mathbf{X}_{t}\right)\right|^{m}}{1+\beta_{c}\left(\mathbf{X}_{t}\right) c_{t}\left(\mathbf{X}_{t}\right)} \frac{\nabla c_{t}\left(\mathbf{X}_{t}\right)}{\left|\nabla c_{t}\left(\mathbf{X}_{t}\right)\right|}
$$

where $c_{t}(\cdot)$ is the actual concentration of the molecular substance (which might evolve according to a proper evolution law, such as a reaction-diffusion $(\mathrm{RD})$ equation) in the place where the test cell passes 
through. For instance, $\alpha_{c}>0$ models the chemical sensitivity of the moving individual, $\beta_{c}>0$ gives saturation effects, while $m>0$ is a Michaelis-Menten-like coefficient. It is straightforward to notice that $m=0$ implies the fact that the chemical gradient determines the direction of the chemotactic velocity and not the modulus. Furthermore, $m=1$ and $\beta_{c}=0$ lead to the most classical form of chemotaxis used in the literature. Actually, if $c$ is a chemoattractant, (2.3) requires a positive sign, if $c$ is instead a chemorepellent, (2.3) requires a negative sign;

- haptotaxis toward regions with higher concentration of ECM, which is implemented by

$$
\mathbf{v}_{d i r}\left(\mathbf{X}_{t}\right)=\alpha_{h}\left(\mathbf{X}_{t}\right) \frac{\left|\nabla h_{t}\left(\mathbf{X}_{t}\right)\right|^{m}}{1+\beta_{h}\left(\mathbf{X}_{t}\right) h_{t}\left(\mathbf{X}_{t}\right)} \frac{\nabla h_{t}\left(\mathbf{X}_{t}\right)}{\left|\nabla h_{t}\left(\mathbf{X}_{t}\right)\right|},
$$

where $h_{t}(\cdot)$ is the concentration of insoluble ECM proteins or of a bound chemical factor and the meaning of $\alpha_{h}, \beta_{h}$ and $m$ is the same as above;

- durotaxis, toward regions with stiffer ECM, given by

$$
\mathbf{v}_{d i r}\left(\mathbf{X}_{t}\right)=\alpha_{e}\left(\mathbf{X}_{t}\right)\left|\nabla e_{t}\left(\mathbf{X}_{t}\right)\right|^{m} \frac{\nabla e_{t}\left(\mathbf{X}_{t}\right)}{\left|\nabla e_{t}\left(\mathbf{X}_{t}\right)\right|}
$$

where $e_{t}(\cdot)$ is a measure of the local matrix stiffness, e.g., the shear modulus.

Of course, a combination of the three above-mentioned effects is also possible. However, a cell preferred velocity may be also determined by selected mechanical interactions occurring with the extracellular environment. In particular, cell migration may be influenced by the mechanical properties of the ECM, capable of initiating series of well-determined mechanotransduction signals within moving individuals (transmitted via transmembrane adhesive molecules and focal points) thereby establishing preferred directions of movement. Preferential velocity fields may finally arise from a non-local (i.e., long-range) environmental sensing experienced by wandering cells.

Cell interaction velocity. The term $\mathbf{v}_{\text {int }}$ represents instead the component of the velocity of the test cell determined by direct intercellular interactions, which can be of various types:

- cell-cell adhesive interactions, which rely upon the expression of cell adhesive molecules (CAMs), e.g., cadherins;

- repulsive interactions, which reproduce cell resistance to compression;

- contact-dependent chemical interactions, i.e., regulated by signaling proteins triggered by cell-cell interactions and able to activate intracellular pathways ultimately mediating cell movement;

- mechanical interactions, such as stresses or shear forces applied to cell membrane.

All these different classes of interactions, which mediate $\mathbf{v}_{\text {int }}$ as a mean field structure, are performed by the test cell $\mathbf{X}_{t}$ through a scanning of the surrounding mass (i.e., of its field cells).

On these considerations, $\mathbf{K}: \mathbb{R}^{n} \times \mathbb{R}^{n} \mapsto \mathbb{R}^{n}$ is indeed an interaction kernel which expresses the direct interaction instances between the test cell $\mathbf{X}_{t}$ and the (infinitesimal) mass $d \mu_{t}(\mathbf{y})$ located in the point $\mathbf{y} \in \mathbb{R}^{n}$. It is natural to assume that the result of the intercellular interactions gives an effect on the direction connecting the interacting individuals, i.e.,

$$
\mathbf{K}\left(\mathbf{X}_{t}, \mathbf{y}\right)=k\left(\left|\mathbf{y}-\mathbf{X}_{t}\right|\right) \frac{\mathbf{y}-\mathbf{X}_{t}}{\left|\mathbf{y}-\mathbf{X}_{t}\right|}
$$

where $k: \mathbb{R} \mapsto \mathbb{R}$ translates the idea that the interactions between the test cell and the surrounding individuals depend on their relative distance and satisfies suitable assumptions of $\mu$-integrability.

The integral in (2.2) has been generally extended over $\mathbb{R}^{n}$ (avoiding cell self-interactions). However, a biologically reasonable assumption is that direct intercellular interactions apply only over a proper interaction neighborhood of the test cell, defined by the compact

$$
U_{\mathbf{X}_{t}}:=\operatorname{supp}\left(k\left(\mathbf{X}_{t}, \cdot\right)\right) \subseteq B_{R}\left(\mathbf{X}_{t}\right),
$$

where $\operatorname{supp}\left(k\left(\mathbf{X}_{t}, \cdot\right)\right)$ defines the support of $k\left(\mathbf{X}_{t}, \cdot\right)$ and $B_{R}\left(\mathbf{X}_{t}\right) \subset \mathbb{R}^{n}$ is the ball centered in $\mathbf{X}_{t}$, whose radius $0<R<+\infty$ represents the sensing radius of the test cell. In this way, the integral in (2.2) can 


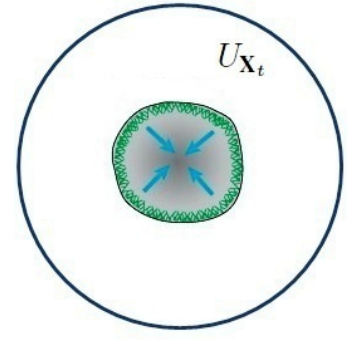

isotropic interactions

$U_{\mathbf{x}_{t}} \equiv B_{R}\left(\mathbf{X}_{t}\right)$

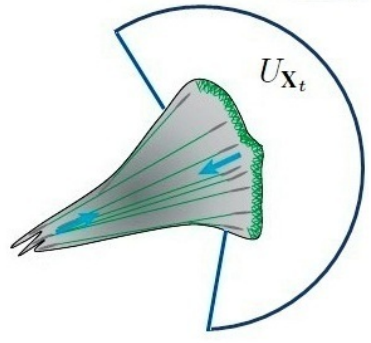

anisotropic interactions

$$
U_{\mathbf{X}_{t}} \subset B_{R}\left(\mathbf{X}_{t}\right)
$$

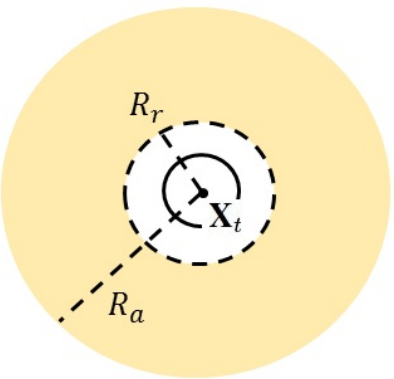

interaction radii

FiguRE 1. Interaction neighborhood of the test cell. Left panel: a round cell is characterized by isotropic interactions (i.e., equally expressed in all directions). Middle panel: a polarized cell is characterized by anisotropic interactions (i.e., expressed only in given directions). In the cell representative images, the arrows represent the tension on the substrate, the thin filaments the expression of cadherin molecules, and the lines the actin filaments. Right panel: in the presented model, each cells is represented as a dimensionless point identified by a random variable $\mathbf{X}_{t}$ that denotes its actual position (black dot). Cell size is taken into account through the repulsive neighborhood, determined by $R_{r}$ (white circle). Such a repulsive radius evaluates in fact the mean cell diameter, whereas the mean cell radius is of course given by $R_{r} / 2$ (black full-line circumference). The maximal extension of cell adhesive protrusions is given by $R_{a}$, which indeed determines the corresponding adhesive neighborhood (colored shadow).

be directly limited on $U_{\mathbf{X}_{t}} \backslash\left\{\mathbf{X}_{t}\right\}$. It is useful to explicitly notice that $U_{\mathbf{X}_{t}}$ may either coincide or be a proper subset of $B_{R}\left(\mathbf{X}_{t}\right)$, see Fig. 1. In the first case, the intercellular interactions are said to be isotropic (i.e., equally expressed all around the test cell). This may apply to cells characterized for instance by a homogeneous distribution of receptors or adhesive molecules all over their membrane. In the second case, interactions are instead anisotropic (i.e., expressed only in specific directions). This mimics the situation either of cells with clusters of transmembrane signaling proteins or of polarized cells able to sense the presence of neighboring individuals only in a specific region of the external space.

Interaction kernel. In the following, we will always assume that the cells within the simulated aggregate are characterized only by isotropic adhesive/repulsive interactions. Indeed, the kernel $\mathbf{K}$ will take the following form:

$$
\begin{aligned}
\mathbf{K}\left(\mathbf{X}_{t}, \mathbf{y}\right) & =\left[k_{r}\left(\left|\mathbf{y}-\mathbf{X}_{t}\right|\right)+k_{a}\left(\left|\mathbf{y}-\mathbf{X}_{t}\right|\right)\right] \frac{\mathbf{y}-\mathbf{X}_{t}}{\left|\mathbf{y}-\mathbf{X}_{t}\right|} \\
& =\left[-\frac{F_{r}}{\left|\mathbf{y}-\mathbf{X}_{t}\right|} 1_{B_{R_{r}}\left(\mathbf{X}_{t}\right)}+F_{a}\left|\mathbf{y}-\mathbf{X}_{t}\right| 1_{B_{R_{a}}\left(\mathbf{X}_{t}\right)}\right] \frac{\mathbf{y}-\mathbf{X}_{t}}{\left|\mathbf{y}-\mathbf{X}_{t}\right|},
\end{aligned}
$$

where $B_{R_{r}}, B_{R_{a}}$ are the isotropic supports of either the repulsive or the adhesive forces, whereas $R_{r}, R_{a}>$ 0 are the relative radii and $F_{r}, F_{a}>0$ the relative strengths. In particular, the form of $\mathbf{K}$ translates the idea that the repulsive and adhesive interactions are, respectively, inversely and directly proportional to the actual distance between interacting individuals. Finally, by fixing the interaction radii, we assume that the intercellular interactions are metric (i.e., within given maximum distances).

Entering in more details in the biological significance of the parameters introduced in (2.8), we can state that (cf. Fig. 1 (right panel)):

- $R_{r}$ can be interpreted as a mean cell diameter, that determines the minimal space occupied by a cell $\left(R_{r} / 2\right.$ evaluates in fact the mean cell radius). We are indeed assuming a short-range repulsive 
interaction, that implements the tendency of most types of cells to stay in an non-compressed state, i.e, to reach a relaxed stress-free configuration;

- $F_{r}$ is an intrinsic cell resistance to compression, that is necessary to allow them to realistically preserve their dimensions and to avoid overlaps;

- $R_{a}$ represents the maximal distance reached by cell mobile adhesive structures (i.e., filopodia, pseudopodia). In this respect, it is consistent to assume $R_{a}>R_{r}$, as wandering cells are able to extend membrane protrusions sufficiently far from their main body;

- $F_{a}$ measures the cell-cell adhesive strength which, from a molecular viewpoint, is regulated by the expression and the activation of specific cadherin molecules.

In conclusion, with the proposed choice for kernel $\mathbf{K}$, the interaction component of the velocity of the test cell reads as:

$$
\mathbf{v}_{\text {int }}\left(\mathbf{X}_{t}\right)=-\int_{B_{R_{r}}\left(\mathbf{X}_{t}\right) \backslash\left\{\mathbf{X}_{t}\right\}} F_{r} \frac{\mathbf{y}-\mathbf{X}_{t}}{\left|\mathbf{y}-\mathbf{X}_{t}\right|^{2}} d \mu_{t}(\mathbf{y})+\int_{B_{R_{a}}\left(\mathbf{X}_{t}\right) \backslash\left\{\mathbf{X}_{t}\right\}} F_{a}\left(\mathbf{y}-\mathbf{X}_{t}\right) d \mu_{t}(\mathbf{y}) .
$$

\subsection{Derivation of the collective mathematical model}

From the phenomenological law of the test cell, it is possible to formally derive an evolution equation for the overall mass measure $\mu_{t}$. As explained in details in [13], the cellular mass, as a physical quantity of cells, has in fact to coherently move with the motion of the single individuals, i.e., we can mathematically say that $\mu_{t}$ is transported by $\mathbf{X}_{t}$. In particular, the following conservation law for the overall cell population can be for instance obtained from (2.2) using Reynolds' theorem (the reader is referred to [13] for the complete procedure):

$$
\frac{\partial \mu_{t}}{\partial t}+\nabla \cdot\left(\mu_{t} \mathbf{v}_{t}\right)=0
$$

where

$$
\mathbf{v}_{t}(\mathbf{x})=\mathbf{v}_{d i r}(\mathbf{x})+\int_{\mathbb{R}^{n} \backslash\{\mathbf{x}\}} \mathbf{K}(\mathbf{x}, \mathbf{y}) d \mu_{t}(\mathbf{y})
$$

is the transport field. A qualitative analysis of the initial-value problem associated with model (2.10)(2.11), concerning in particular the existence of measure-valued solutions and the convergence to them of a properly conceived approximation scheme, is performed in [44].

It is useful to remark that Eq. (2.10) provides a collective-Eulerian representation of the dynamics of the overall cell population, with the individual trajectory of the test cell blurred into the spatio-temporal evolution of the mass distribution, represented by the measure $\mu_{t}$. In other words, the variable $\mathbf{X}_{t}$ represents the individuality of a generic representative cell of the population, whereas the mass measure $\mu_{t}$ represents the collectivity of the cell ensemble. We finally underline that the overall collective dynamics can be defined as spontaneous and self-emergent. The model in fact prescribes the phenomenological behavior only of single cells that, because of their limited interaction field (i.e., $U_{\mathbf{X}_{t}}$ ), are not able to influence the evolution of distant individuals.

\subsection{Specification of the mass measure $\mu_{t}$}

The above-presented approach is expected to provide consistent mathematical tools to address the multiscale issues posed by biological problems. In fact, the description of the system in terms of an abstract measure does not force an a priori choice of the scale of representation, which can then be a posteriori deferred only to the specific structure given to $\mu_{t}$.

Discrete description. A microscopic/discrete description of the cellular aggregate (and of its evolution), in which each cell is singularly represented, can be achieved by using an atomic mass measure constituted by a sum of Dirac masses centered in each cell position as,

$$
\mu_{t}(E)=\sum_{i=1}^{N} \delta_{\mathbf{x}_{t}^{i}}(E):=m_{t}(E) \quad \forall E \in \mathcal{B}\left(\mathbb{R}^{n}\right),
$$


where the $\left\{\mathbf{x}_{t}^{i}\right\}_{i=1}^{N}$ are the space positions where the $N$ cells forming the aggregate are located at time $t$. Plugging (2.12) in (2.2), the phenomenological model specializes as

$$
\dot{\mathbf{X}}_{t}=\mathbf{v}_{d i r}\left(\mathbf{X}_{t}\right)+\sum_{\substack{i=1 \\ \mathbf{x}_{t}^{i} \neq \mathbf{x}_{t}}}^{N} \mathbf{K}\left(\mathbf{X}_{t}, \mathbf{x}_{t}^{i}\right)
$$

while, plugging the same relation in (2.10), we obtain that the collective evolution of the cellular ensemble is described by the following system:

$$
\left\{\begin{array}{l}
\frac{d \mathbf{x}_{t}^{i}}{d t}=\mathbf{v}_{t}\left(\mathbf{x}_{t}^{i}\right) \\
\mathbf{v}_{t}\left(\mathbf{x}_{t}^{i}\right)=\mathbf{v}_{d i r}\left(\mathbf{x}_{t}^{i}\right)+\sum_{\substack{j=1 \\
j \neq i}}^{N} \mathbf{K}\left(\mathbf{x}_{t}^{i}, \mathbf{x}_{t}^{j}\right),
\end{array}\right.
$$

for all $i=1, \ldots, N$.

Continuous description. A macroscopic/continuous description of the dynamical system, in which the cell population is represented through a spatial density, requires to assume that the mass measure is absolutely continuous with respect to the $d$-dimensional Lebesgue measure $\mathcal{L}^{d}$ (i.e., $\mu_{t} \ll \mathcal{L}^{d}$ ):

$$
\mu_{t}(E)=\int_{E} \rho_{t}(\mathbf{y}) d \mathbf{y}:=M_{t}(E) \quad \forall E \in \mathcal{B}\left(\mathbb{R}^{n}\right),
$$

where $\rho_{t}(\cdot):[0, T] \times \mathbb{R}^{n} \mapsto[0,+\infty), T>0$ being a certain final time (possibly $+\infty$ ), is the numerical space density of the cell population at time $t$, whose existence is asserted by the Radon-Nikodym's Theorem. Hence, the phenomenological model takes the form:

$$
\dot{\mathbf{X}}_{t}=\mathbf{v}_{d i r}\left(\mathbf{X}_{t}\right)+\int_{\mathbb{R}^{n} \backslash\left\{\mathbf{X}_{t}\right\}} \mathbf{K}\left(\mathbf{X}_{t}, \mathbf{y}\right) \rho_{t}(\mathbf{y}) d \mathbf{y},
$$

whereas the overall cellular aggregate evolves following

$$
\left\{\begin{array}{l}
\frac{\partial \rho_{t}(\mathbf{x})}{\partial t}+\nabla \cdot\left(\rho_{t}(\mathbf{x}) \mathbf{v}_{t}(\mathbf{x})\right)=0 \\
\mathbf{v}_{t}(\mathbf{x})=\mathbf{v}_{d i r}(\mathbf{x})+\int_{\mathbb{R}^{n} \backslash\{\mathbf{x}\}} \mathbf{K}(\mathbf{x}, \mathbf{y}) \rho_{t}(\mathbf{y}) d \mathbf{y} .
\end{array}\right.
$$

Multiscale description. It is finally possible to provide a multiscale-hybrid description of the cellular system, i.e., neither completely discrete nor completely continuous, which is able to account for a proper amount of microscopic granularity within the macroscopic flow of cells. This amounts to choose the measure $\mu_{t}$ as a linear combination of the discrete and the continuous description of the system, i.e.,

$$
\mu_{t}(E)=(1-u) m_{t}(E)+u M_{t}(E) \quad \forall E \in \mathcal{B}\left(\mathbb{R}^{n}\right) .
$$

In particular, $u \in[0,1]$ is a multiscale coupling parameter which determines the relative importance given to each type of representation, i.e., the percent mass, with another view point, shifted from the microscopic to the macroscopic scale as a consequence of the multiscale coupling. For instance, it is possible, keeping the same cellular mass (i.e., the same number of cells), to switch from a more discrete/microscopic description to a more distributed/continuous representation of the system by only tuning the value of $u$. It is worth stressing that when $u \neq 0,1$ neither the sole set of points $\left\{\mathbf{x}_{t}^{i}\right\}_{i=1}^{N}$ (i.e., the microscopic scale) 
nor the sole density $\rho_{t}$ (i.e., the macroscopic scale) provides the actual space distribution of the cells. According to Eq. (2.18), the complete picture results instead from a $u$-weighted joint contribution of the twos. The relative phenomenological model therefore reads as:

$$
\dot{\mathbf{X}}_{t}=\mathbf{v}_{d i r}\left(\mathbf{X}_{t}\right)+(1-u) \sum_{\substack{i=1 \\ \mathbf{x}_{t}^{i} \neq \mathbf{x}_{t}}}^{N} \mathbf{K}\left(\mathbf{X}_{t}, \mathbf{x}_{t}^{i}\right)+u \int_{\mathbb{R}^{n} \backslash\left\{\mathbf{X}_{t}\right\}} \mathbf{K}\left(\mathbf{X}_{t}, \mathbf{y}\right) \rho_{t}(\mathbf{y}) d \mathbf{y} .
$$

In this case, plugging (2.18) in the measure conservation equation (2.10), it is straightforward to formally obtain a combination of Eqs. (2.14) and (2.17), where the discrete and continuous descriptive instances of the cellular mass are separately transported by construction, being coupled through the same velocity field:

$$
\left\{\begin{array}{l}
\frac{d \mathbf{x}_{t}^{i}}{d t}=\mathbf{v}_{t}\left(\mathbf{x}_{t}^{i}\right) \quad \forall i=1, \ldots, N \\
\frac{\partial \rho_{t}(\mathbf{x})}{\partial t}+\nabla \cdot\left(\rho_{t}(\mathbf{x}) \mathbf{v}_{t}(\mathbf{x})\right)=0 \\
\mathbf{v}_{t}(\mathbf{x})=\mathbf{v}_{d i r}(\mathbf{x})+(1-u) \sum_{\substack{j=1 \\
\mathbf{x}_{t}^{j} \neq \mathbf{x}}}^{N} \mathbf{K}\left(\mathbf{x}, \mathbf{x}_{t}^{j}\right)+u \int_{\mathbb{R}^{n} \backslash\{\mathbf{x}\}} \mathbf{K}(\mathbf{x}, \mathbf{y}) \rho_{t}(\mathbf{y}) d \mathbf{y} .
\end{array}\right.
$$

In particular, the interaction component of the velocity coincides neither with the fully microscopic nor with the fully macroscopic one. This definitely makes the overall dynamics not a simple superposition of the individual microscopic and macroscopic dynamics. It is in fact worth noticing that the point $\mathbf{x}$ may or may not be one of the positions of a single cell. In more details, computing $\mathbf{v}_{t}(\mathbf{x})$ for $\mathbf{x}=\mathbf{x}_{t}^{i}$ shows that the interaction velocity of the $i$-th cells accounts not only for the other cells microscopically represented but also for those cells represented as an undifferentiated density. Analogously, computing $\mathbf{v}_{t}(\mathbf{x})$ for $\mathbf{x}$ different from all the $\mathbf{x}_{t}^{i}$ 's shows that the interaction velocity of an infinitesimal reference cell volume centered in $\mathbf{x}$ depends not only on the density distribution but also on the microscopic cells, which play the role of singularities in the average cellular distribution.

\subsection{Dimensionless form}

In order to identify the relevant dimensionless parameters and to correctly scale the microscopic and macroscopic contributions, it is convenient to derive a non-dimensional form of the evolution equations for both the discrete and the continuous counterparts of the simulated system. For this purpose, let's scale lengths with the repulsion radius $R_{r}$, velocities with a characteristic velocity $V$ to be specified later on, times with $\frac{R_{r}}{V}$ and number densities with a characteristic density $\frac{1}{R_{r}^{n}}$. Dimensionless time, space, and velocities are denoted with *. In particular

- $t^{*}=\frac{V}{R_{r}} t, \quad \mathbf{x}^{*}=\frac{\mathbf{x}}{R_{r}}, \quad \mathbf{X}_{t^{*}}^{*}=\frac{1}{R_{r}} \mathbf{X}_{\frac{R_{r}}{V} t^{*}}, \quad \mathbf{x}_{t^{*}}^{* i}=\frac{1}{R_{r}}\left(\mathbf{x}_{\frac{R_{r}}{V} t^{*}}^{i}\right) \quad \forall i=1, \ldots, N ;$

- $\mathbf{v}_{d i r}^{*}=\frac{1}{V} \mathbf{v}_{d i r}, \quad \mathbf{v}_{i n t}^{*}=\frac{1}{V} \mathbf{v}_{i n t}, \quad \mathbf{K}^{*}\left(\mathbf{x}^{*}, \mathbf{y}^{*}\right)=\frac{1}{V} \mathbf{K}\left(R_{r} \mathbf{x}^{*}, R_{r} \mathbf{y}^{*}\right)$;

- $\rho_{t^{*}}^{*}\left(\mathbf{x}^{*}\right)=\frac{1}{R_{r}^{n}} \rho_{\frac{R_{r}}{V} t^{*}}\left(R_{r} \mathbf{x}^{*}\right)$.

The non-dimensional differential mass measure $d \mu_{t}^{*}$, in the general multiscale form, is:

$$
\begin{aligned}
& d \mu_{t^{*}}^{*}\left(\mathbf{x}^{*}\right)=d \mu_{\frac{R_{r}}{V} t^{*}}\left(R_{r} \mathbf{x}^{*}\right)=(1-u) \sum_{i} \delta_{R_{r} \mathbf{x}_{t^{*}}^{* i}}\left(R_{r} \mathbf{x}^{*}\right)+u \rho_{\frac{R_{r}}{V} t^{*}}\left(R_{r} \mathbf{x}^{*}\right) d\left(R_{r} \mathbf{x}^{*}\right)= \\
& =(1-u) \sum_{i} \delta_{\mathbf{x}_{t^{*}}^{* i}}\left(\mathbf{x}^{*}\right)+u \rho_{t^{*}}^{*}\left(\mathbf{x}^{*}\right) d \mathbf{x}^{*}=(1-u) m_{t^{*}}^{*}\left(\mathbf{x}^{*}\right)+u d M_{t^{*}}^{*}\left(\mathbf{x}^{*}\right),
\end{aligned}
$$


where $m_{t^{*}}^{*}$ and $M_{t^{*}}^{*}$ are the dimensionless discrete and continuous descriptions of the cellular mass. It is now trivial to obtain the non-dimensional form of the equations regulating the dynamics of the two descriptive instances of the cellular mass:

$$
\left\{\begin{array}{l}
\frac{\partial \rho_{t^{*}}^{*}\left(\mathbf{x}^{*}\right)}{\partial t^{*}}+\nabla^{*} \cdot\left(\rho_{t^{*}}^{*}\left(\mathbf{x}^{*}\right) \mathbf{v}_{t^{*}}^{*}\left(\mathbf{x}^{*}\right)\right)=0 \\
\frac{d \mathbf{x}_{t^{*}}^{* i}}{d t^{*}}=\mathbf{v}_{t^{*}}^{*}\left(\mathbf{x}_{t^{*}}^{* i}\right), \quad i=1, \ldots, N
\end{array}\right.
$$

Finally, the natural velocity scale stems out from the non-dimensional form of the interaction component of the transport velocity, where the interaction kernel is defined as in Eq. (2.8). In fact, chosen $V=\frac{F_{r}}{R_{r}}$, it can be written as:

$$
\begin{aligned}
& \mathbf{v}_{i n t}^{*}\left(\mathbf{x}^{*}\right)=-\left[(1-u) \sum_{\substack{j=1 \\
\mathbf{x}^{* j} \in\left(B_{1}\right) \mathbf{x}^{*} \\
\mathbf{x}^{* j} \neq \mathbf{x}^{*}}}^{N} \frac{\left(\mathbf{x}^{* j}-\mathbf{x}^{*}\right)}{\left|\mathbf{x}^{* j}-\mathbf{x}^{*}\right|^{2}}+u \int_{\left(B_{1}\right)_{\mathbf{x}^{*}} \backslash\left\{\mathbf{x}^{*}\right\}} \frac{\left(\mathbf{y}^{*}-\mathbf{x}^{*}\right)}{\left|\mathbf{y}^{*}-\mathbf{x}^{*}\right|^{2}} \rho^{*} d \mathbf{y}^{*}\right] \\
& +F^{*}\left[(1-u) \sum_{\substack{j=1 \\
\mathbf{x}^{* j} \in\left(B_{\left.R^{*}\right)}\right)_{\mathbf{x}^{*}} \\
\mathbf{x}^{* j} \neq \mathbf{x}^{*}}}^{N}\left(\mathbf{x}^{* j}-\mathbf{x}^{*}\right)+u \int_{\left(B_{R^{*}}\right)_{\mathbf{x}^{*}} \backslash\left\{\mathbf{x}^{*}\right\}}\left(\mathbf{y}^{*}-\mathbf{x}^{*}\right) \rho^{*} d \mathbf{y}^{*}\right] \text {, }
\end{aligned}
$$

where

$$
F^{*}=\frac{F_{a}}{F_{r}} R_{r}^{2}
$$

and

$$
\left\{\begin{array}{l}
\left(B_{1}\right) \mathbf{X}_{t^{*}}^{*}=\left\{\mathbf{y}^{*}:\left|\mathbf{y}^{*}-\mathbf{X}_{t^{*}}^{*}\right|<1\right\} ; \\
\left(B_{R^{*}}\right)_{\mathbf{X}_{t^{*}}^{*}}=\left\{\mathbf{y}^{*}:\left|\mathbf{y}^{*}-\mathbf{X}_{t^{*}}^{*}\right|<\frac{R_{a}}{R_{r}}=R^{*}\right\} .
\end{array}\right.
$$

Indeed, the space of all possible variations of the four dimensional model parameters can be efficiently covered by varying the two non-dimensional parameters, i.e., $F^{*}$ and $R^{*}$. In particular, $R^{*}>1$ since, as previously stated, $R_{a}>R_{r}$.

\section{Results}

\subsection{Simulation Details}

In this section, we perform numerical simulations produced by a modified version of the numerical scheme developed in [14] (see also [35,44] for error and convergence analysis), specifically designed for the measuretheoretic framework with hybrid $\mu_{t}$. In particular, we invariably refer to and solve the non-dimensional form of the equations (cf. Sec. 2.4).

In all realizations, we deal with two-dimensional $(n=2)$ square bounded domains $\Omega \subset \mathbb{R}^{2}$ (whose size may vary in the different cases, according to specific dynamics), with no flux conditions on the border of the square. This means that the cellular mass can not flow out of $\Omega$, therefore being conserved. $\Omega$ may indeed represent a section of a Petri dish, typically used for in vitro biological/biomedical assays.

Cell dynamics are completely determined by the interaction component of their velocity (i.e., $\mathbf{v}_{d i r}=0$ in Eq. (2.2)). The parameters introduced in the interaction kernel (2.8), describing the biophysical properties of the cells, can be evaluated consistently with the biological considerations clarified along the text. In particular, given that the repulsive radius is fixed equal to $20 \mu \mathrm{m}$ (i.e., the mean measure of most eukaryotic cells [1]) and that the cell attractive one (i.e., the maximal extension of their filopods) 


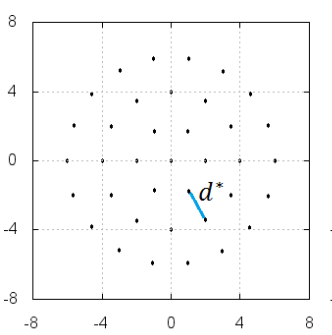

(a) $u=0$

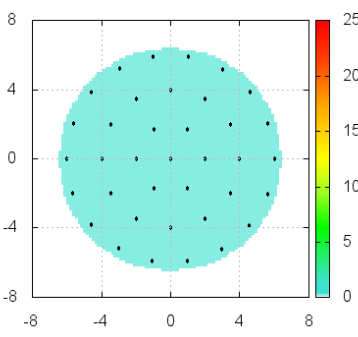

(b) $u \neq\{0 ; 1\}$

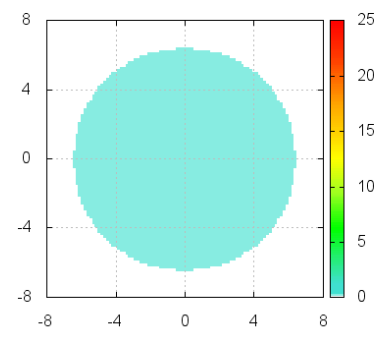

(c) $u=1$

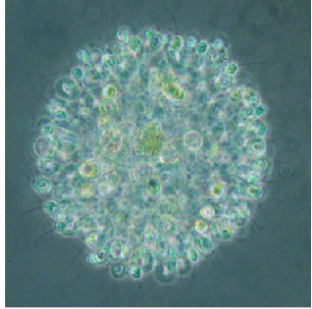

(d) experimental image

FIgURE 2. For illustrative purposes, the initial condition of most simulations: a round and spatially homogeneous colony of $N=37$ cells is placed in the center of the domain. At the microscopic level, the individuals are arranged with a given radial distance, (a) panel. The relative continuous distribution is then computed according to Eq. (3.1), (bc) panels. Such an initial configuration resembles an experimental cell aggregate cultured in vitro, (d) panel. The image of the colony of human umbilical vein endothelial cells (HUVECs) is a courtesy of the Institute of Cancer Research and Treatment of Candiolo.

can reach $60 \mu \mathrm{m}$ [1], the dimensionless radius $R^{*}$ is kept fixed to 3. On the opposite, the non-dimensional force $F^{*}$ will change to reproduce different quantities of expressed CAMs (we recall that $F^{*} \propto F_{a}$ ).

The initial conditions of all simulations consist of a centered round colony of $N=37$ cells, as shown in Fig. 2. In particular, for the microscopic description, the single individuals are arranged with a fixed initial radial distance $d^{*}=d / R_{r}$. The coherent initial density is indeed computed according to a local average of the microscopic mass distribution, as

$$
\rho_{0}^{*}\left(\mathbf{x}^{*}\right)= \begin{cases}\frac{37}{\pi\left(R_{0}^{*}\right)^{2}} & \text { if } \mathbf{x}^{*} \in B_{R_{0}^{*}} ; \\ 0 & \text { else, }\end{cases}
$$

where $B_{R_{0}^{*}} \subset \mathbb{R}^{n}$ is the ball centered in the middle of the domain and the initial dimensionless radius is $R_{0}^{*}=3 d^{*}+1 / 2$ (i.e., $R_{0}=R_{0}^{*} R_{r}=3 d^{*} R_{r}+R_{r} / 2$ where the second term at the r.h.s. accounts for the finite radius of the external cells). Such a configuration reproduces a round cell colony experimentally cultured, see panel (d) in Fig. 2.

The specific value given to the initial intercellular distance define the initial stress conditions of the aggregate. In this respect, by figuring out a purely microscopic description of the system, we can distinguish three situations, see Fig. 3:

1. for $d^{*} \leq 1$ (i.e., $d \leq R_{r}<R_{a}$ ), the colony is compressed, as the cells are too close to each other. Indeed, they will initially undergo both repulsive and adhesive interactions;

2. for $1<d^{*} \leq R^{*}=3$ (i.e., $R_{r}<d \leq R_{a}$ ), the cellular ensemble is in a relaxed non-compressed state and the component individuals initially experience only adhesive interactions;

3. for $d^{*}>R^{*}=3$ (i.e., $R_{r}<R_{a}<d$ ), the cells are too far to sense each other and therefore to interact. In this respect, the initial configuration of the system reproduces a dispersed cell population rather than a compact colony.

By further considering binary intercellular interactions (which represent of course a simplified scheme of the general case where intercellular interactions occur between several cells), we are allowed also to speculate on the equilibrium cell configurations that would emerge from the different initial conditions:

1. the initial coexistence of both adhesion and compression results in an equilibrium configuration determined by an intercellular distance $d_{e q}^{*}$ such that

$$
-\frac{1}{d_{e q}^{*}}+F^{*} d_{e q}^{*}=0
$$




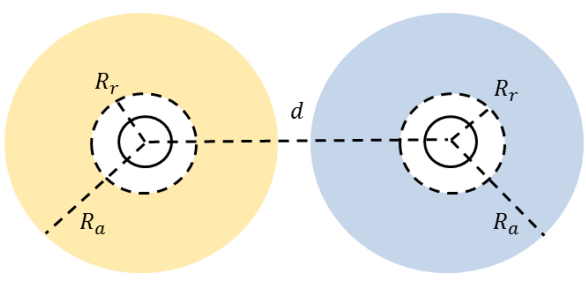

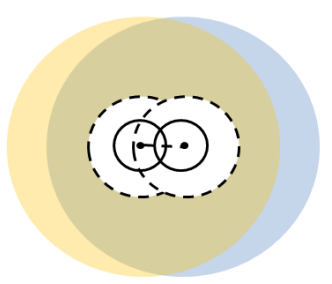

$d \leq R_{r}<R_{a}$

$d^{*} \leq 1$

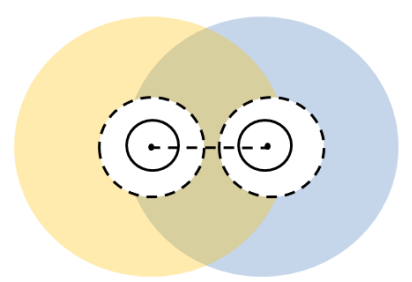

$R_{r}<d \leq R_{a}$

$1<d^{*} \leq R^{*}$

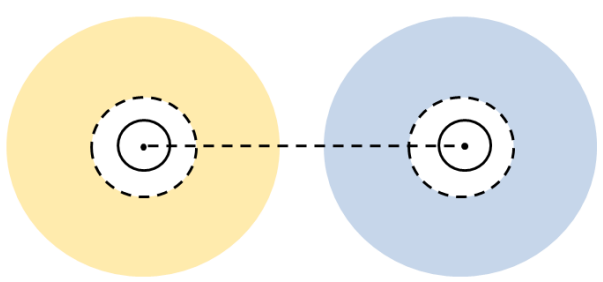

$R_{r}<R_{a}<d$

$d^{*}>R^{*}$

FIgURE 3. Top panel: the distance between two individuals is evaluated by the distance between their actual location (dashed line). Bottom panels: different values of the initial intercellular distance $d$ correspond to different cell stress conditions. In particular, if $d \leq R_{r}$, cells are compressed since their bodies overlap. On the contrary, if $d \in\left(R_{r} ; R_{a}\right]$, cells are in a relaxed state and interact only with adhesive forces. Finally, if $d>R_{a}$, cells are dispersed. Dashed circumferences represents cell body, see Fig. 1 (right panel).

which gives $d_{e q}^{*}=\frac{1}{\sqrt{F^{*}}}$. This implies that, at the equilibrium, cell extension is characterized by a radius of $1 /\left(2 \sqrt{F^{*}}\right)$;

2. if initially only adhesive interactions are in force, the cells start aggregating until repulsive interactions are activated. Then, the dynamics follow that presented in the previous case;

3 . in the case of a scattered cell population, the initial condition represents a steady configuration of the system. It is indeed not possible to draw conclusions on cell radius in equilibrium conditions, except from the fact that cell size has to belong to $\left[1, R^{*}\right]$.

To quantify the effects of the different model parameters, and the interconnection between the different descriptive scales, we concentrate on the moment of inertia of the colony around its center of mass $\left(\mathbf{x}_{t^{*}}^{G}\right)^{*}=\frac{1}{\mu_{t^{*}}^{*}\left(\Omega^{*}\right)} \int_{\Omega^{*}} \mathbf{x}^{*} d \mu_{t^{*}}^{*}\left(\mathbf{x}^{*}\right)$ that writes:

$$
I_{t^{*}}^{G}=\int_{\Omega^{*}}\left|\mathbf{x}^{*}-\left(\mathbf{x}_{t^{*}}^{G}\right)^{*}\right|^{2} d \mu_{t^{*}}^{*}\left(\mathbf{x}^{*}\right)
$$

The value of $I_{t^{*}}^{G}$, as known from classical mechanics, provides in fact quantitative information on the evolution of the global distribution of the cellular mass around its center of mass. Given a radius of a colony, lower values of $I_{t^{*}}^{G}$ correspond in fact to denser configurations around the center of mass. In addition, $I_{t^{*}}^{G}$ gives quantitative evaluations of expansion/shrinking processes. For instance, decreasing values of $I_{t^{*}}^{G}$ correspond to aggregating cell colonies, while increasing values of $I_{t^{*}}^{G}$ refer to scattering cell populations. It is useful to remark that, by replacing in the above formulas $\mu_{t^{*}}^{*}$ with either the discrete, the continuous or the hybrid determinations, it is possible to study the moment of inertia of the relative descriptive instance of the system. In particular, due to Eq. (2.18), the moment of inertia of the hybrid measure is a linear combination of the moments of inertia of both the microscopic and the macroscopic masses. 


\subsection{Varying the intercellular interaction force $F^{*}$}

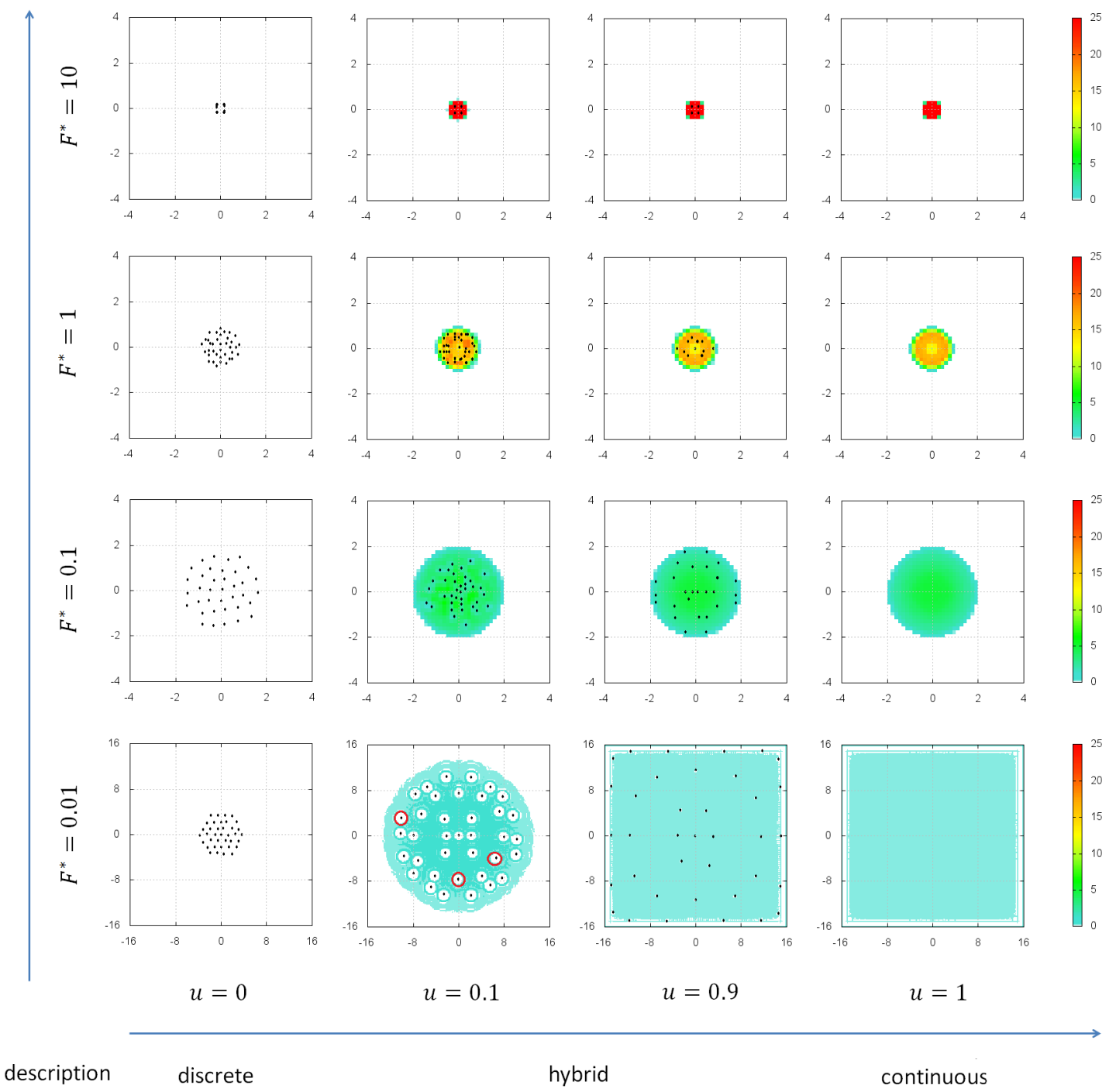

Figure 4. Final configuration (i.e., at $T^{*}$ such that $F^{*} T^{*}=1$ ) of the cellular aggregate for different values of the non-dimensional intercellular interaction force $F^{*}$, as captured by different descriptive approaches. In all cases, the initial radial microscopic intercellular distance is $d^{*}=2$, so that the initial radius of the colony is $R_{0}^{*}=6.5$, whereas the dimensionless radius of the intercellular interactions is $R^{*}=3$. For the reader's convenience, in the panel corresponding to $F^{*}=0.01$ and $u=0.1$, we drew circles with radius equal to the non-dimensional repulsion radius around some discrete points that actually represent cells with a finite size.

In this first set of simulations, we investigate how the evolution of the cell colony is influenced by variations of the non-dimensional interaction force $F^{*}$. In particular, given its definition in Eq. (2.24), increments in $F^{*}$ correspond to increments in the ratio of the cell-cell adhesive interactions with respect to the repulsive interactions. The results obtained by the different mathematical descriptions of the cellular ensemble, i.e., for $u=0,0.1,0.9,1$ are then compared. In all cases, the initial microscopic intercellular 


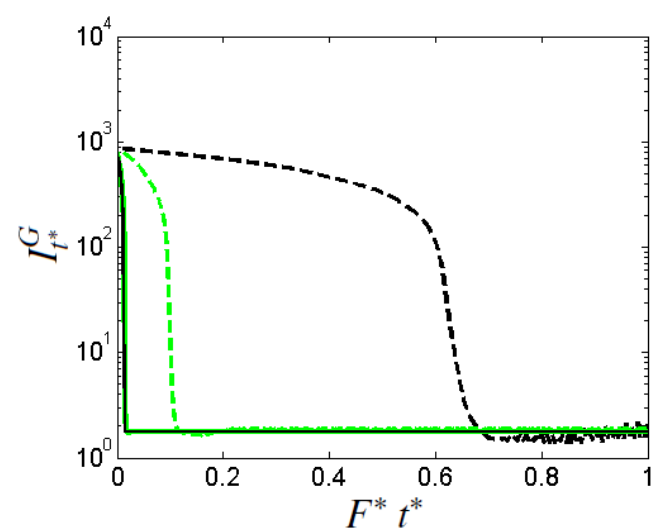

(a) $F^{*}=10$

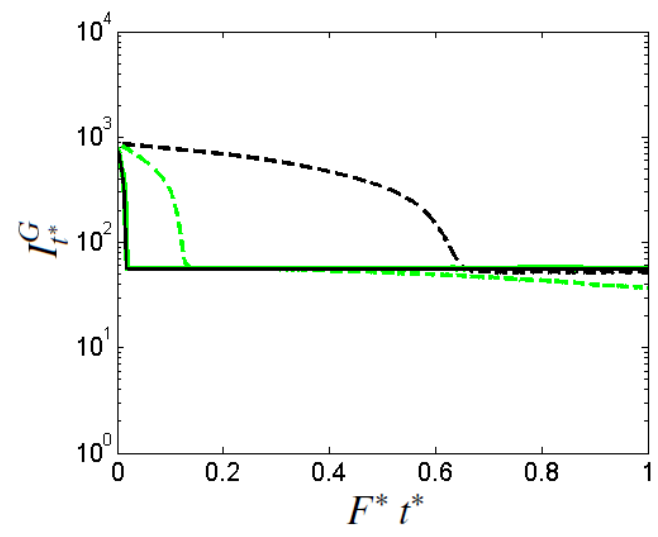

(c) $F^{*}=0.1$

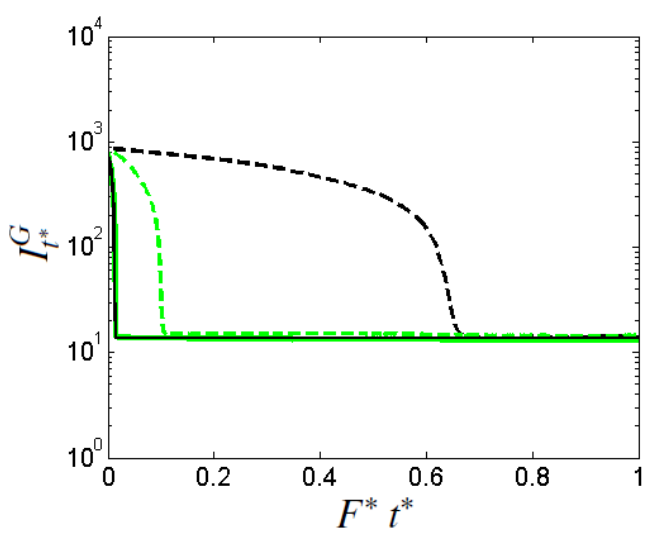

(b) $F^{*}=1$

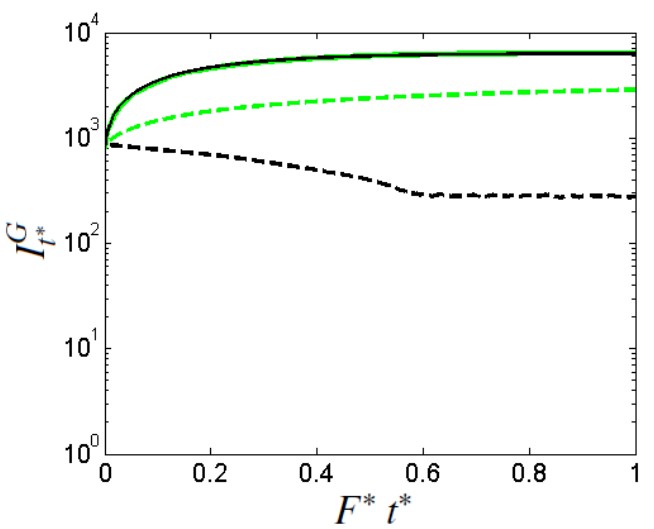

(d) $F^{*}=0.01$

FiguRE 5. Evolution in time of the baricentrical moment of inertia $I_{t^{*}}^{G}$ for the different values of $F^{*}$, as captured by different descriptive approaches: $u=0$ (dark dashed line), $u=0.1$ (light dashed line), $u=0.9$ (light full line), and $u=1$ (dark full line).

distance is $d^{*}=2$ so that the initial radius of the colony is $R_{0}^{*}=6.5$. It is worth noticing that such a value of $d^{*}$ establishes a relaxed configuration of the colony, indeed initially characterized by only intercellular adhesive interactions (i.e., the above-mentioned case (2)). In order to provide a coherent comparison between the system dynamics for the different values of $F^{*}$, the final observation times set chosen such that $F^{*} T^{*}=1$.

At $F^{*}=0.1$, see Fig. 4 (third row), the cell colony starts aggregating until stabilizing in an equilibrium configuration, defined by a round cluster with a diameter of almost 4 . This final state is characterized by a balance between adhesive and repulsive forces, so that the aggregate does not significantly evolve any longer. Such an equilibrium configuration is caught almost at all scales. The only exception is for $u=0.1$ : in this case, the moment of inertia in fact continues to slightly decrease reaching later on an equilibrium value $\approx 25$ (at about $F^{*} t^{*} \approx 2$ ). The explanation resides in the fact that, while the continuous distribution reaches the steady state, the discrete individuals keep moving and slightly approach the center of the colony. Their equilibrium configuration is in fact perturbed by the even small continuous contribution, that encourages them to constantly reorganize.

At higher $F^{*}$ (i.e., $=1 ; 10$ ), for all values of $u$, the aggregate quickly compresses in a very small cluster, see Fig. 4 (top and second row) and Fig. 5 (a-b). This is the consequence of the fact that the adhesive strength, overcoming the repulsive one, forces the cells to be closely packed. In particular, the diameter of 
the final colony decreases with increments in $F^{*}$. It is worth noticing however that too compressed final clusters (although representing equilibrium configurations arising from the specific parameter settings) can not be considered realistic, as biological cells need a minimal space to survive. This means that the adhesive force is unrealistically high compared to the value of the repulsion force.

Interestingly, all the final states of the cellular mass encountered so far have been obtained by all types of description: however, the transient dynamics are slightly different. In particular, as captured by the plots of the evolution of $I_{t^{*}}^{G}$, increments in the discrete contribution (i.e., decrements in $u$ ) result in slower aggregations, see Fig. 5 (a-c). A possible explanation is that the more the description of the system is microscopically-oriented, the less the intercellular force is distributed throughout the colony, that therefore takes more time to completely aggregate.

Finally, at a low value of $F^{*}=0.01$, significant differences in the system dynamics are observed at the different scales, see Fig. 4 (bottom row). For $u=0$, the starting configuration is almost the steady one: because the repulsive force is able to overcome the intercellular adhesiveness, the single individuals starts aggregating but, when they come too close, they repel each other and stabilize to a distance almost equal to 1 (the value of the non-dimensional repulsion radius, see Eq. (2.25)). Adding a small continuous contribution to the discrete setting (i.e., $u=0.1$ ) the evolution of the system dramatically changes. The cell colony in fact radially expands towards the border of the domain, with some kind of round "density holes", encircled in red for the reader's convenience in the relative panel of Fig. 4, around every microscopic cells. These areas of very low continuous density, which have a radius close to $R_{r}^{*}$ (i.e., 1), are caused by the microscopic repulsion that, in this neighborhood, has a greater impact than adhesion at the macroscopic level, so that the continuous density is repelled (recall that the microscopic cell distribution is seen as a sum of singularities within the macroscopic cell density). At even higher contributions of the continuous description (i.e., $u=0.9$ ), the aggregate expands out to fill the domain, because of the prominent role played by macroscopic repulsion. Furthermore, the non-negligible granular effects (i.e., the density holes) of the previous case are no longer obtained. In this sense, we can say that the contribution of the macroscopic scale has a homogenizing effect on the overall dynamics, which are analogous to the purely continuous case (i.e., $u=1$ ). With a biological view point, it is obvious that in this case only the discrete-microscopic approach captures a realistic evolution of the aggregate. It is useful to notice that the asymptotic behavior of $I_{t^{*}}^{G}$ described in Fig. 5 (d) is due to the no flux condition imposed at the boundary of the domain.

In Fig. 10 (top graph), we collect the final moments of inertia, evaluated for the different descriptive instances of the cellular mass, for several values of $F^{*}$ (i.e., both the previously introduced ones and some others). It can be noticed that, for high values of $F^{*}$ (i.e., greater than $\approx 0.1$ ), no significant discrepancy is observed regardless of the distinct scale used to reproduce the system. This means that coherent (but not always realistic, see the comments above and in the figure) results can be obtained by describing the cellular ensemble with the different approaches. On the opposite, at low values of $F^{*}$ (i.e., lower than $\approx$ 0.1 ), different scales of description lead to different outcomes. In particular, a purely continuous dynamics is much more robust to discrete perturbations (see the almost perfect superposition between dark and light full lines) than a purely discrete one with respect to continuous perturbations (see the significant discrepancy between dark and light dashed lines). In this case only a fully microscopic approach is able to reproduce a realistic evolution of the colony.

\subsection{Varying the initial intercellular distance $d^{*}$}

We next turn to analyze how the initial configuration of the aggregate affects its subsequent dynamics. In particular, we maintain a homogeneous and round cell distribution, but we vary the initial radial intercellular distance $d^{*}$ (i.e., which results in different initial diameters of the colony and therefore in different stress conditions, as previously stated), see Fig. 6. In all this set of realizations, the nondimensional interaction force $F^{*}$ is taken equal to 0.1 , that is the value characteristic of the biologically reasonable equilibrium state found in the previous section. In this respect, it is useful to remark that larger values of $F^{*}$ result in unrealistically packed cell colonies, whereas significantly smaller values of $F^{*}$ cause dramatic expansions of the aggregate in the case of macroscopic or quasi-macroscopic dynamics. For reader's convenience we here recall that the dimensionless interaction radius $R^{*}$ is always taken equal to 3 . 


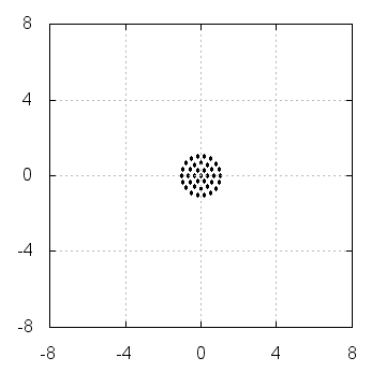

(a) $d^{*}=0.35$

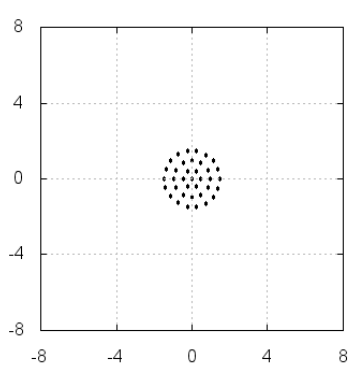

(b) $d^{*}=0.5$

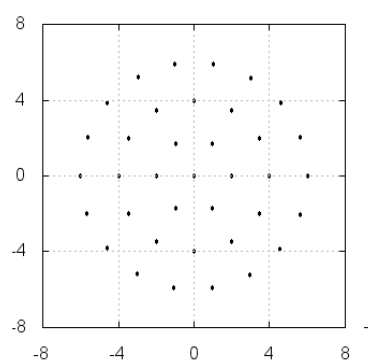

(c) $d^{*}=2$

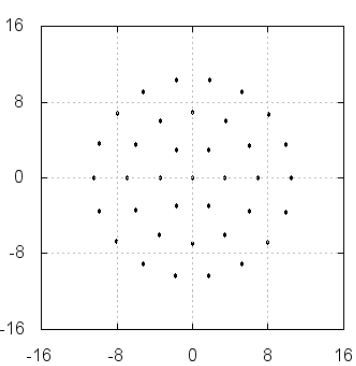

(d) $d^{*}=3.5$

FiguRE 6. Microscopic (i.e., at $u=0$ ) initial configuration of the aggregate for different values of the initial intercellular distance $d^{*}$. In particular, the initial dimensionless radii of the colony are: (a) $R_{0}^{*}=1.55$, (b) $R_{0}^{*}=2$, (c) $R_{0}^{*}=6.5$, and (d) $R_{0}^{*}=11$. In particular, (a) corresponds to an initial aggregate compression, both (b) and (c) refer to an initially relaxed colony while (d), at the microscopic level, no longer results in a single aggregate but in a dispersed population of non-interacting cells. In each case, the coherent homogeneous initial density $\rho_{0}^{*}$ is indeed computed according to Eq. (3.1).

If the cellular ensemble is initially compressed (i.e., $d^{*}=0.35$, so that $R_{0}^{*}=1.55$ ), it quickly expands and then stabilizes in the equilibrium configuration previously displayed, i.e., a round cluster with an dimensionless diameter of 4, see Fig. 7 (bottom row) and the graph in Fig. 8 (d). This is due to the fact that the aggregate is initially subjected to a sort of internal pressure, where the repulsive force plays a predominant role. However, as soon as the cells are distant enough, intercellular repulsion is balanced by adhesive interactions and the system stabilizes. In particular, such a dynamics is captured by all the different scales of description.

Moderate increments in $d^{*}$ result in initial non-compressed configurations of the cell aggregate, which indeed does not undergo any relaxation mechanisms (see Sec. 3.1). In particular, if the initial intracellular distance $d^{*}$ is taken equal to 0.5 (such as $R_{0}^{*}$ becomes 2), the initial condition of the colony is almost the equilibrium one. Indeed, the aggregate does not significantly evolve, for all $u$, as shown in Fig. 7 (third row) and Fig. 8 (c).

For sufficiently higher values of $d^{*}$ (i.e., $=2$, so that $R_{0}^{*}=6.5$ ), the colony instead aggregates, however reaching again the equilibrium morphology. The aggregate shrinkage, already obtained in the previous section (see the third row of Fig. 4), is due to the adhesive forces, which initially act on the cells and which are subsequently balanced by the intercellular repulsion. In this respect, the initial condition of the system can be interpreted as colony formed by adhesive cells that however are too extended and therefore tend to assume a more compact ensemble configuration. Such a behavior is obtained by all types of mathematical description (with only a small discrepancy for $u=0.1$, as previously commented), see Fig. 7 (second row) and the plot of $I_{t^{*}}^{G}$ in Fig. 8 (b).

At even larger $d^{*}$ (i.e., $=3.5$, such as $R_{0}^{*}=11$ ), important differences arise from the distinct approach used to represent the cellular ensemble, as reproduced in the top panels of Fig. 7. In the purely discrete case, the starting configuration is a steady one. As the initial intercellular distance is larger than the cell sensing radius, the individual cells are not able to feel the presence of their neighbors and therefore they do not move at all: in this respect, as previously stated, the initial configuration of the system reproduces a scattered cell populations of non-interacting individuals, rather than a single cell colony. Adding a small continuous contribution to the discrete setting (i.e., $u=0.1$ ) changes dramatically the dynamics with respect to the purely discrete case. The mass in fact slowly concentrates, and finally stabilizes, in four clusters: this is due to the "fragility" of the purely discrete dynamics, which strongly depend on the precise locations of the cells. An even small spatially continuous component of the measure $\mu_{t}$ allows the cells to feel, within their sensing neighborhood, the presence/influence of the surrounding field individuals besides their exact position, eventually resulting in a qualitatively very different evolution of the system. Actually, the continuous distribution acts as a "communicator" between the discrete entities otherwise too far to aggregate. Conversely, a purely continuous description (i.e., $u=1$ ) triggers an unrealistic 

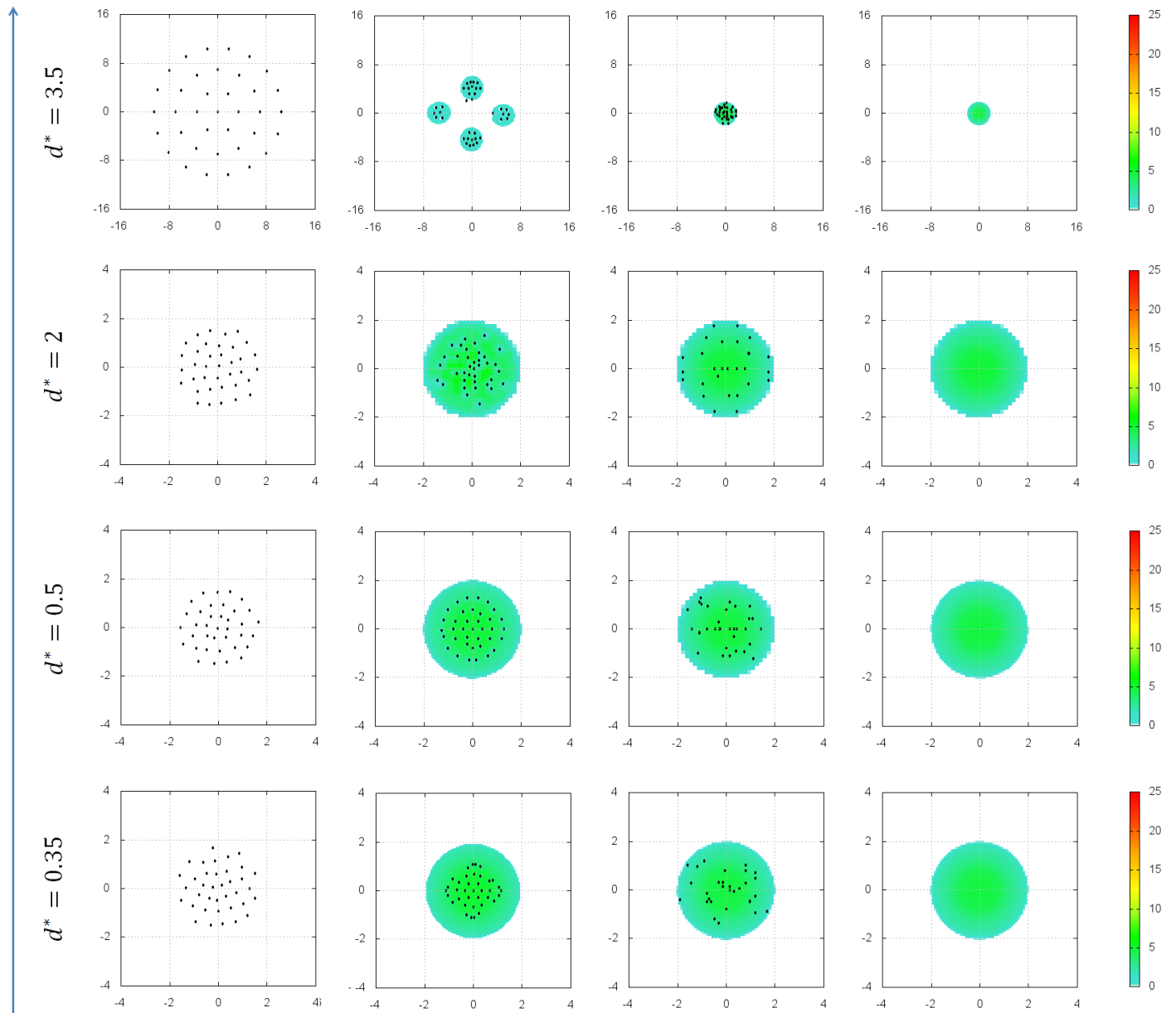

$u=0$

$u=0.1$

$u=0.9$

$u=1$

description

discrete

hybrid

continuous

FiguRE 7. Final configuration (i.e., at $T^{*}=10$ such that $F^{*} T^{*}=1$, as $F^{*}$ is set equal to 0.1 ) of the cellular aggregate for different values of the initial radial intercellular distance $d^{*}$ (i.e., whose dimensional value is $d=d^{*} R_{r}$ ), as captured by different descriptive approaches. In all cases, the dimensionless radius of the intercellular interactions is $R^{*}=3$.

complete aggregation of the colony, which collapses toward the equilibrium configuration. In particular, a small discrete contribution to the continuous setting (i.e., $u=0.9$ ) does not appreciably perturb the outcome with respect to the fully macroscopic case. Interestingly, as captured by the time-lapse images in Fig. 9, as well as by the plots of the evolution of the baricentrical moment of inertia in Fig. 8 (a), the transient dynamics for both $u=1$ and $u=0.9$ display a step-wise behavior. In fact at $t^{*}=1$ (i.e., $F^{*} t^{*}=0.1$ ), the cellular aggregate seems to quickly stabilize in an intermediate four-cluster configuration qualitatively similar to the equilibrium configuration obtained for $u=0.1$. However, in contrast with the case with $u=0.1$, the enhanced influence of the continuous dynamics allows a further aggregation, that results in the single final and stationary cluster.

In Fig. 10 (bottom graph), we represent the final the moment of inertia $I_{T^{*}}^{G}$ for different values of $d^{*}$ (some of which are in addition to the previously introduced ones). We can indeed observe that, 


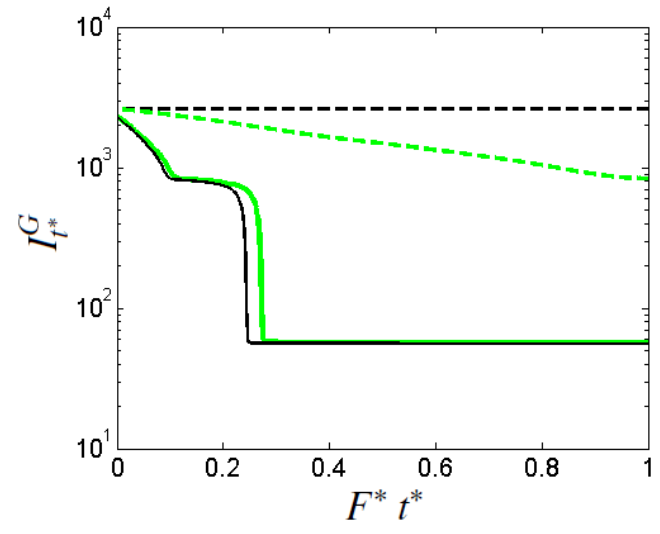

(a) $d^{*}=3.5$

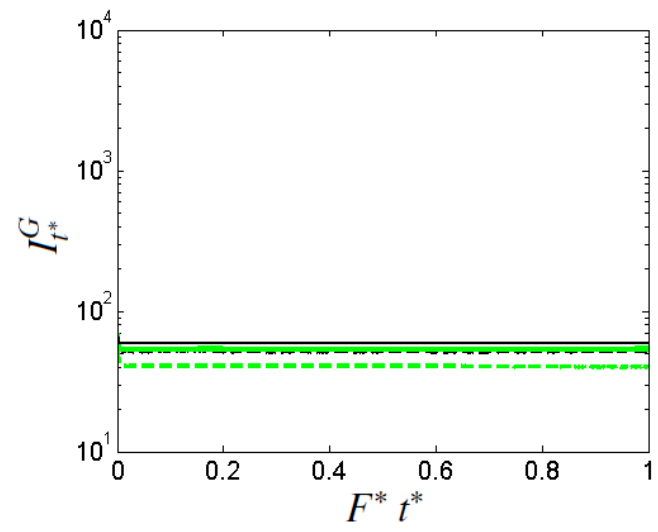

(c) $d^{*}=0.5$

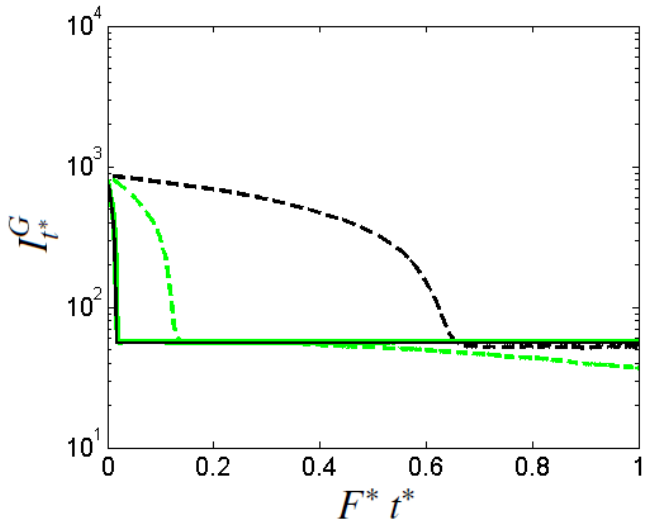

(b) $d^{*}=2$

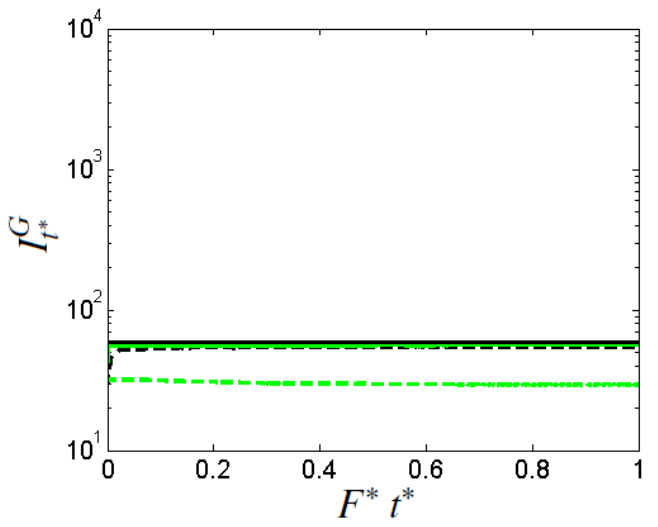

(d) $d^{*}=0.35$

Figure 8. Evolution in time of the baricentical moment of inertia $I_{t^{*}}^{G}$ for the different values of $d^{*}$, as captured by different descriptive approaches: $u=0$ (dark dashed line), $u=0.1$ (light dashed line), $u=0.9$ (light full line), and $u=1$ (dark full line). To have a coherent comparison in the different cases, we plot $I_{t^{*}}^{G}$ vs. $F^{*} t^{*}$, until $T^{*}=10$ such that $F^{*} T^{*}=1$, as $F^{*}$ is set equal to 0.1 .

for initially compressed colonies (i.e., $d^{*} \leq 1$ ), the equilibrium configuration represents the final state of evolution of the colony, regardless of the scale used for its mathematical description. Such model dynamics also hold for initially relaxed aggregates that are characterized by an initial intercellular distance $d^{*}$ up to $\approx 2$. Then, for initially relaxed colonies characterized by greater initial intercellular distances, a discrepancy in the evolution of the system occurs between the purely discrete description and all the other representations. Finally, as previously observed, in the case of an initially dispersed population of cells (i.e., $d^{*}>3$ ), the dynamics completely arise from the mathematical description chosen. In particular, a discrete-microscopic view point is the only one able to capture a realistic behavior of the cell population.

Summing up, we can state that in a specific range of the model parameters, i.e., high dimensionless interaction forces and low dimensionless initial intercellular distances, all the mathematical types of description (discrete, continuous or hybrid) give almost the same evolution, as well as the final configuration, of the biological system. On the opposite, outside this set of values, the dynamics of the simulated system mainly arise from the scale used for its representation, in particular relying on the presence or the absence of a continuous contribution. It is then useful to remark that the model outcomes in the case of $F^{*} \approx 1$ or 

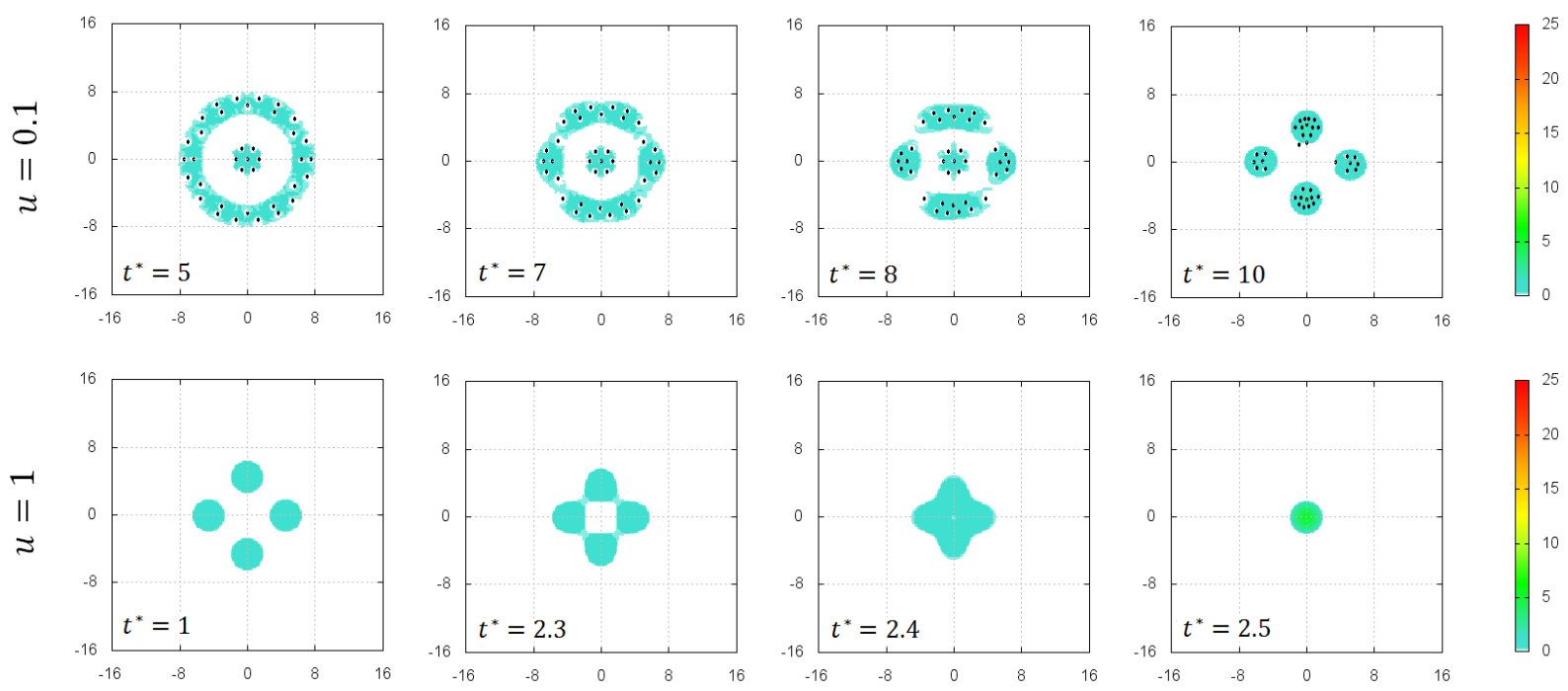

Figure 9. Evolution of the colony in the case of $d^{*}=3.5$, for $u=0.1$ (top panels) and $u=1$ (bottom panels). The four cluster-configuration is a steady one when the microscopic scale plays a dominant role. On the opposite, a further aggregation occurs when the description of the system is biased toward a macroscopic/continuous point of view.

higher, although consistent across the different mathematical descriptions, are not biologically realistic. This is also the case of both the hybrid and the purely macroscopic dynamics obtained for an initially dispersed cell aggregate (i.e., $d^{*}>3$ ). Finally, we underline that we have not precisely determined the parameter threshold values characterizing the transition between coherent and discrepant model dynamics and between realistic and unrealistic model outcomes, as a more extensive sensitivity analysis would have fallen outside the purpose of this work. Nevertheless, we are in the position of speculating that such exact threshold values would not significantly differ from those found out with our dissertation.

\section{Model extension to multiple cell populations}

In most biological systems, multiple cell populations, or multiple differentiated clones of the same cell line, can coexist. For example, non-healthy organs and tissues contain both damaged and normal cells. Similarly, leukocytes, erythrocyte, and platelets are all transported by blood flow. In particular, each cell type is characterized by a different behavior, different endogenous/exogenous interactions and, possibly, different levels of activity. An extension of the proposed model in this respect is therefore necessary in order to increase its realism and the number (and the detail) of possible applications.

Recalling the discussion presented in the previous sections, the dynamics of a test cell of a population $p$, where $p=1, \ldots, P$ and $P$ is the total number of populations taken into account (each of them with a constant and finite number of individuals $N_{p}$ ), follows the proper extension of the standard law (2.2):

$$
\dot{\mathbf{X}}_{t, p}=\mathbf{v}_{d i r}\left(\mathbf{X}_{t, p}\right)+\mathbf{v}_{i n t}^{p}\left(\mathbf{X}_{t, p}\right)=\mathbf{v}_{d i r}\left(\mathbf{X}_{t, p}\right)+\sum_{q=1}^{P} \int_{\mathbb{R}^{n} \backslash\left\{\mathbf{X}_{t, p}\right\}} \mathbf{K}_{p q}\left(\mathbf{X}_{t, p}, \mathbf{y}\right) d \mu_{t, q}(\mathbf{y}) .
$$

$\mathbf{v}_{\text {dir }}$ is, as previously stated, a preferential velocity characteristic of cell $\mathbf{X}_{t, p}$ (that may be in common for all individuals of the same population), whereas $\mathbf{v}_{i n t}^{p}$ represents the interaction component of its velocity which, in this model extension, is determined by the sum of the contributions of both endogenous 

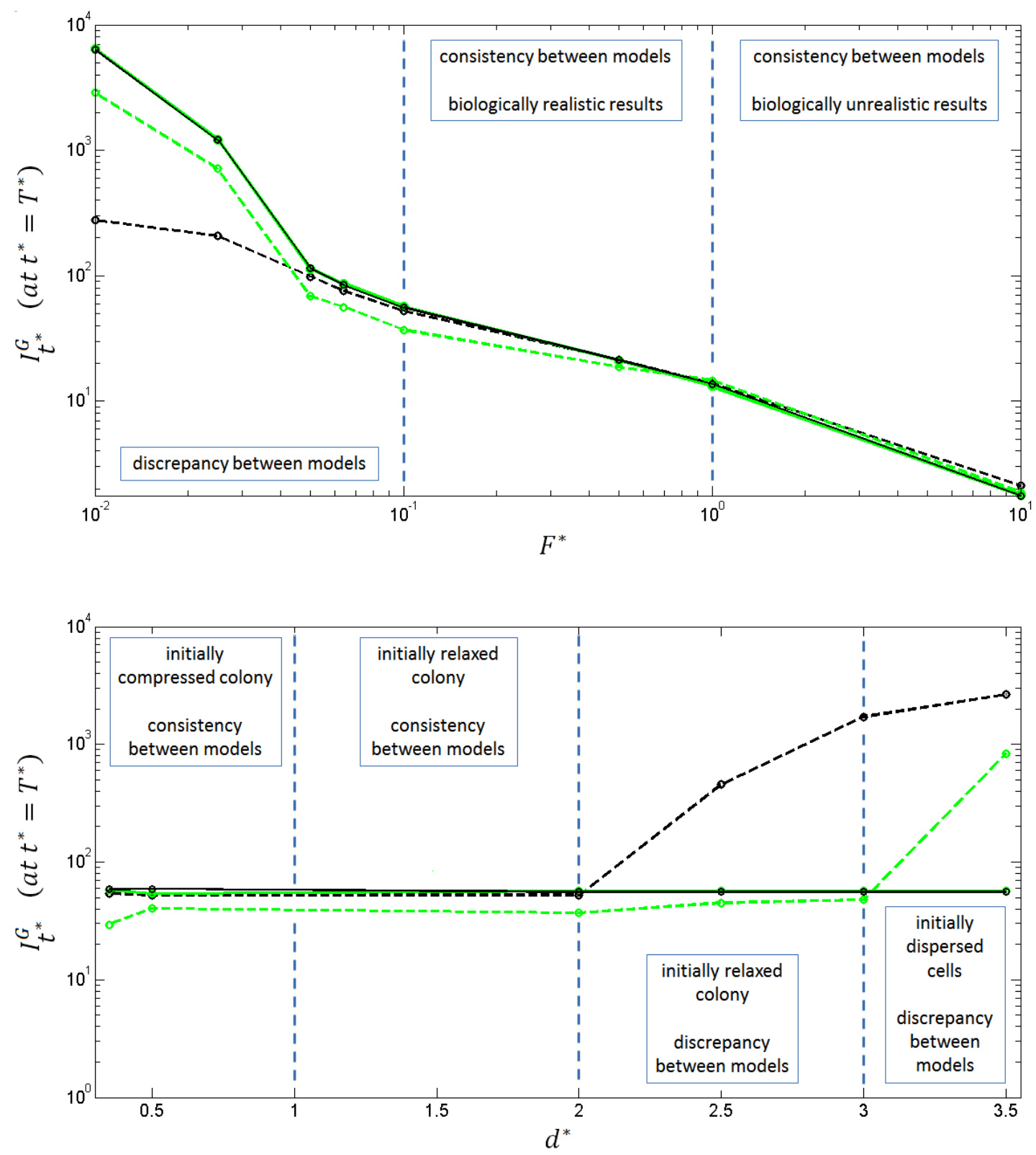

FiguRE 10. Summary of the final moment of inertia (i.e., at $T^{*}$ such that $F^{*} T^{*}=1$ ) obtained by varying either the adimensional intercellular force $F^{*}$ (top panel) or the dimensionless intercellular initial distance $d^{*}$ (bottom panel), as obtained by the different descriptive approaches: $u=0$ (dark dashed line), $u=0.1$ (light dashed line), $u=0.9$ (light full line), and $u=1$ (dark full line).

(i.e., between individuals of the same population) and exogenous (i.e., between individuals of different populations) intercellular interactions. It is useful to notice that the form of all interaction kernels $\mathbf{K}_{p q}$ is again defined by Eqs. (2.6)-(2.8). At this regard, the right way to interpret the specific subscripts is the following: the generic interaction term $\mathbf{K}_{p q}$ defines how an individual of population $p$ is influenced in its dynamics by an individual belonging to population $q$. 
Finally, the evolution equation, as long as the transport velocity, of the mass measure of each population, obtained with the same procedure previously-proposed, results as:

$$
\left\{\begin{array}{l}
\frac{\partial \mu_{t, p}}{\partial t}+\nabla \cdot\left(\mu_{t, p} \mathbf{v}_{t, p}\right)=0, \quad p=1, \ldots, P \\
\mathbf{v}_{t, p}(\mathbf{x})=\mathbf{v}_{d i r}(\mathbf{x})+\sum_{q=1}^{P} \int_{\mathbb{R}^{n} \backslash\{\mathbf{x}\}} \mathbf{K}_{p q}(\mathbf{x}, \mathbf{y}) d \mu_{t, q}(\mathbf{y}) .
\end{array}\right.
$$

Following the considerations presented in Sec. 2.3, each population can be finally characterized by a purely discrete (cf. (2.12)), a purely continuous (cf. (2.15)), or a hybrid description (cf. (2.18), with $u_{p} \in[0,1]$ for all $\left.p=1 \ldots, P\right)$.

\section{A specific case: few activated discrete cells and an undifferentiated continuous aggregate}

As seen, the extended modeling framework allows to use within the same environment a different type of description for each population. For instance, this is of particular relevance in those processes where few specialized and activated leader cells need to be properly described by a concentrated (i.e., discrete) distribution, whereas non-specialized and inactivated individuals can be represented, in average, as a continuously distributed group with a low individual level of detail. In these cases, the discrete-continuous coupling is expected to determine the formation of patterns, as the continuous aggregates are collectively guided by the small number of discrete differentiated individuals. This is what happens in a wide range of collective cell migrations, which characterize for example regeneration of tissues [47], physiopathological blood vessel formation $[6,9,10]$ and metastatic infiltration of solid tumors $[24,27,41,48]$ (we refer the interested reader also to [25,28], where differentiated cell behavior driving collective patterning is extensively reviewed). To analyze these aspects and to provide relevant illustrative simulations, we focus on the evolution of a cell aggregate which is differentiated in two different clones, however belonging to the same cell lineage:

1. population $p=1$ of $N_{1}$ activated individuals, that are suitably described with a microscopic-discrete view point (i.e., $\left.\mu_{t, 1}(E)=\sum_{i=1}^{N_{1}} \delta_{\mathbf{x}_{t, 1}^{i}}(E)\right)$. They have their own preferred/directional velocity, e.g., due to the chemotactic sensitivity and interact with both cellular types;

2. population $p=2$ of $N_{2}$ non-differentiated cells, that are indeed represented with a macroscopic continuous approximation (i.e., $\left.\mu_{t, 2}(E)=\int_{E} \rho_{t, 2}(\mathbf{y}) d \mathbf{y}\right)$. They only experience intercellular interactions with both cellular types.

Taking into account the analytic architecture presented so far, the evolution of the whole biological environment is determined by a system of coupled discrete-continuous equations which, in the nondimensional form, reads as:

$$
\left\{\begin{array}{l}
\frac{d \mathbf{x}_{t^{*}, 1}^{* i}}{d t^{*}}=\mathbf{v}_{t^{*}, 1}^{*}\left(\mathbf{x}_{t^{*}, 1}^{* i}\right) \quad \forall i=1, \ldots, N_{1} \\
\frac{\partial \rho_{t^{*}, 2}^{*}\left(\mathbf{x}^{*}\right)}{\partial t^{*}}+\nabla^{*} \cdot\left(\rho_{t^{*}, 2}^{*}\left(\mathbf{x}^{*}\right) \mathbf{v}_{t^{*}, 2}^{*}\left(\mathbf{x}^{*}\right)\right)=0
\end{array}\right.
$$


where the specific velocity fields of the two populations are

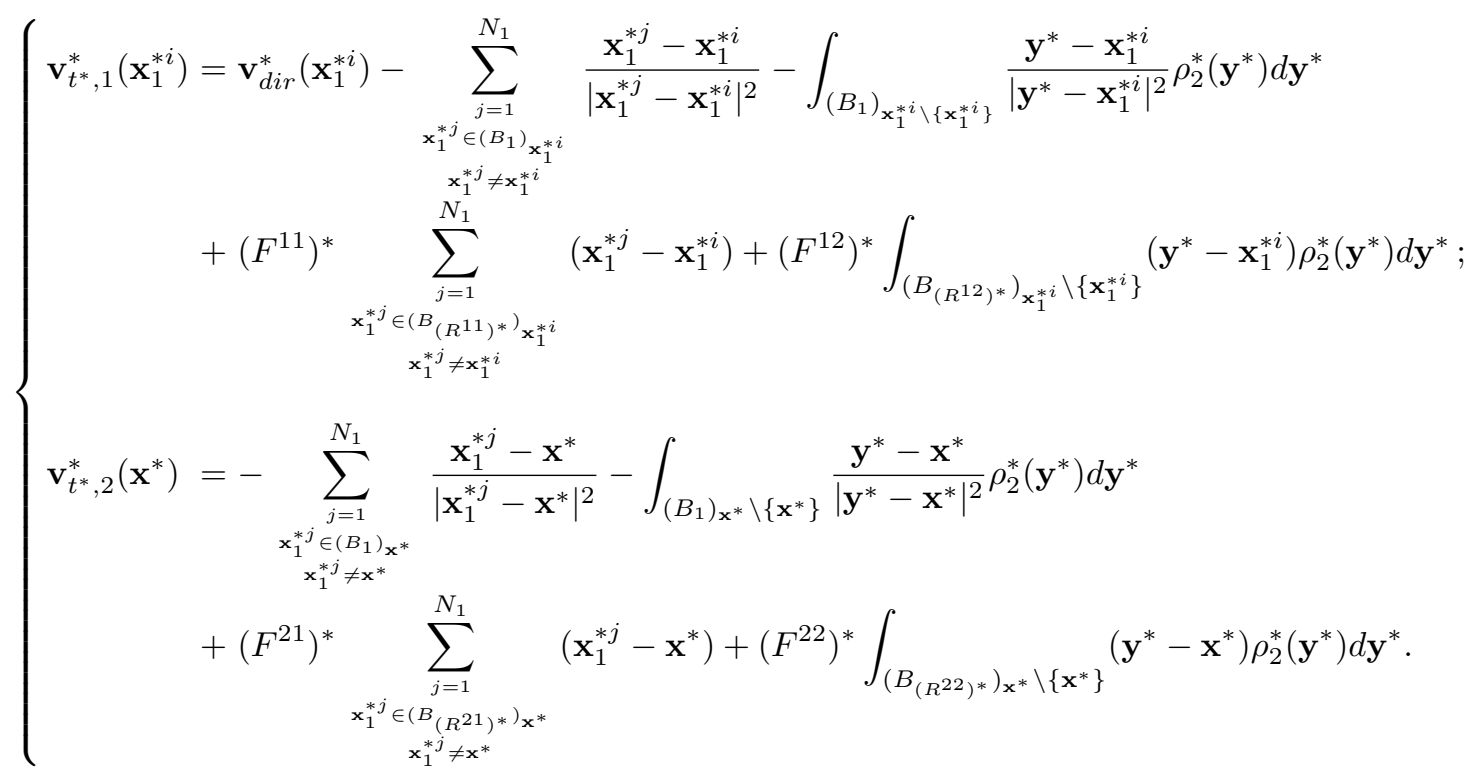

Coherently with the case of a single population, for each $p, q \in\{1,2\}$, the non-dimensional intracellular interaction force is defined as $\left(F^{p q}\right)^{*}=\frac{F_{a}^{p q}}{F_{r}} R_{r}^{2}$ whereas the relative non-dimensional interaction radius is $\left(R^{p q}\right)^{*}=\frac{R_{a}^{p q}}{R_{r}}$ where, for all $p, q \in\{1,2\}, R_{r}=R_{r}^{p q}$ and $F_{r}=F_{r}^{p q}$ represent the common cell dimension and intrinsic resistance to compression. This assumption is consistent with the fact that we are dealing with two populations belonging to the same cell line. In particular, $R_{r}$ is maintained equal to $20 \mu \mathrm{m}$, as in the single population case.

A detailed analytical analysis of the presented hybrid discrete-continuous environment is performed in [13]. In particular, that work inquires the similarities and the differences (evaluated in terms of an a priori stability estimate) in the dynamics of the two-population system, which may derive from an $a$ priori choice of a discrete or of a continuous representation for the non-activated cells (i.e., of population 2).

\subsection{Simulation details}

We deal again with two-dimensional bounded domains $\Omega^{*} \subset \mathbb{R}^{2}$ (whose size will vary according to the dynamics of interest), with no flux conditions. In all cases, the initial configuration consists in few differentiated cells (i.e., belonging to population 1) placed on the border of a homogenous round colony of inactivated individuals (i.e., belonging to population 2), which has a non-dimensional initial radius $R_{0}^{*}=2$ (i.e., $R_{0}=R_{r} R_{0}^{*}=40 \mu \mathrm{m}$ ). The number of cells of the continuous colony is again fixed equal to 37, so that the initial density is defined by Eq. (3.1). This type of initial condition may reproduce a round aggregate of tumor cells with an external ring of highly metabolic metastatic individuals and an inner core of quiescent cells, or an endothelial/epithelial cell system where few tip individuals are able to behave as guidance leaders for migration for the rest of the stalk/follower mass, see Fig. 11.

The values of the interaction radii are determined according to biological considerations. First, since filopods of activated cells are typically more able to wander in the neighboring space than those of inactivated individuals, it is consistent to assume $R_{a}^{11}>R_{a}^{22}$, and therefore $\left(R^{11}\right)^{*}>\left(R^{22}\right)^{*}$. Then, it is realistic to estimate the radii of exogenous interactions as the intermediate measure, i.e., $R_{a}^{12}=R_{a}^{21}=$ $\left(R_{a}^{11}+R_{a}^{22}\right) / 2$ and $\left(R^{12}\right)^{*}=\left(R^{21}\right)^{*}=\left(\left(R^{11}\right)^{*}+\left(R^{22}\right)^{*}\right) / 2$. Assuming that the maximal filopod extension is nearly $60 \mu \mathrm{m}$ (cf. Sec. 3.1), a plausible matrix of the non-dimensional interaction radii is

$$
\mathbf{R}^{*}=\left(\begin{array}{cc}
3 & 2.25 \\
2.25 & 1.5
\end{array}\right)
$$




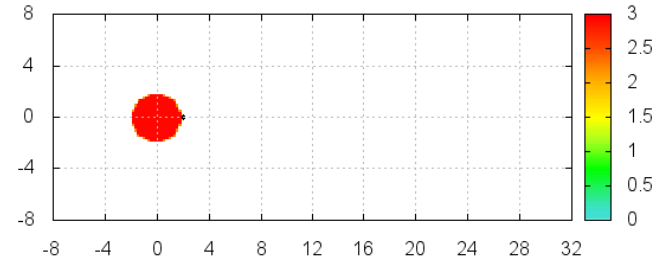

(a)

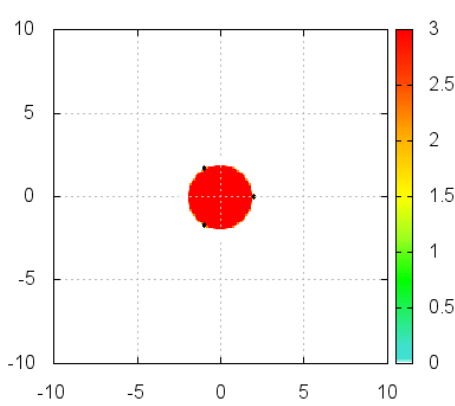

(b)

FiguRE 11. Initial conditions of the hybrid biological system. A round and spatially homogeneous colony of $N_{2}=37$ undifferentiated cells, described with a continuum approximation, coexists with one, (a) panel, or three, (b) panel, activated individuals, which are discretely modeled. It is useful to remark that the two populations are assumed to be different clones of the same cell line (i.e., they have analogous dimensions and resistance to compression). These configurations may reproduce a tumor spheroid characterized by both metastatic and quiescent cells or an endothelial cell colony with few tip/leader individuals and a stalk/follower aggregate.

A proper non-dimensional value of the endogenous interaction force of population $2,\left(F^{22}\right)^{*}=0.4$, is established, after a parametric analysis, to guarantee the equilibrium of the continuum aggregate in the absence of exogenous interactions. We then set a low $\left(F^{11}\right)^{*}=0.1$ to avoid significative mutual influences between activated individuals. The values of the exogenous interaction forces will be instead discussed in the following.

The preferred velocity of the discrete cells, whose specific form will be given in the next sections, reproduces the directional movement of activated individuals towards zones with higher amounts of motility factors, such as insoluble extracellular matrix proteins (fibrin, vitronectin, and some of the collagen family) or diffusible chemicals (nutrients and growth factors).

\subsection{An activated cell with an undifferentiated aggregate}

We first investigate the case of a single differentiated cell (Fig. 11 (a)) with a constant horizontal velocity $\mathbf{v}_{\text {dir }}^{*}\left(\mathbf{x}_{1}^{* 1}\right)=v_{0} \mathbf{i}$, where $\mathbf{i}$ is the unit vector in the $\mathrm{x}$-direction. Recalling that cells belonging to population 2 only experience intercellular interactions, we assume that they are particularly attracted by the activated individual, i.e., $\left(F^{21}\right)^{*}>\left(F^{22}\right)^{*}$. On the opposite, the discrete cell is supposed to interact in the same way with any other individual, regardless of the population it belongs to, since its dynamics are mainly determined by the directional component of the velocity. Indeed we set $\left(F^{12}\right)^{*}=\left(F^{11}\right)^{*}$. The resulting matrix of the nondimensional interaction forces then reads as:

$$
\mathbf{F}_{1}^{*}=\left(\begin{array}{cc}
0.1 & 0.1 \\
1 & 0.4
\end{array}\right)
$$

In particular, the specific value of $\left(F^{21}\right)^{*}$ has been chosen to have a good balance between a dramatic repulsion and a unrealistically absorption of the differentiated cell within the continuous aggregate. This is confirmed by the fact that, in the absence of any preferred velocity (i.e., $v_{0}=0$ ), the proposed parameter setting gives rise only to plausible cell reorganization phenomena, as the undifferentiated colony reaches an inner equilibrium and the discrete cell remains at its border, as shown in Fig. 12 (a).

For low values of the non-dimensional preferred velocity (say $v_{0} \leq 20$ ), the continuous aggregate, after reorganizing towards the stable configuration, undergoes a rigid translation following the discrete cell motion, as shown by the snapshots reported in Fig. 12 (b). Finally, for higher values of $v_{0}$, the undifferentiated colony, after the usual self-remodeling, is unable to follow the activated cell, that moves away, as reproduced in Fig. 12 (c). This behavior is due to the fact that the magnitude of the exogenous 

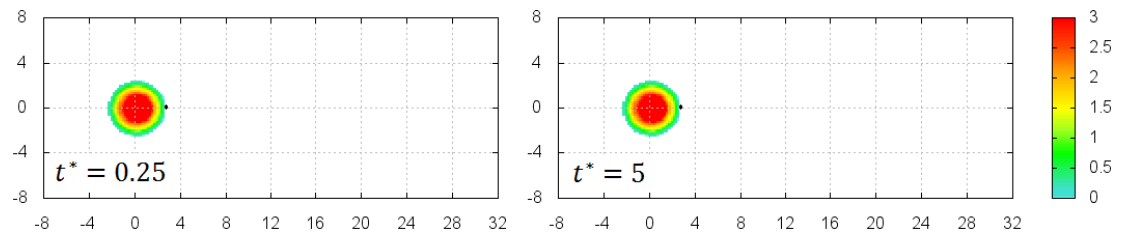

(a) $v_{0}=0$
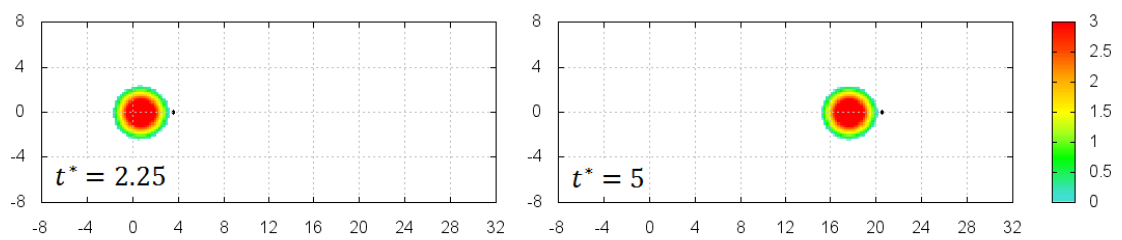

(b) $v_{0}=20$
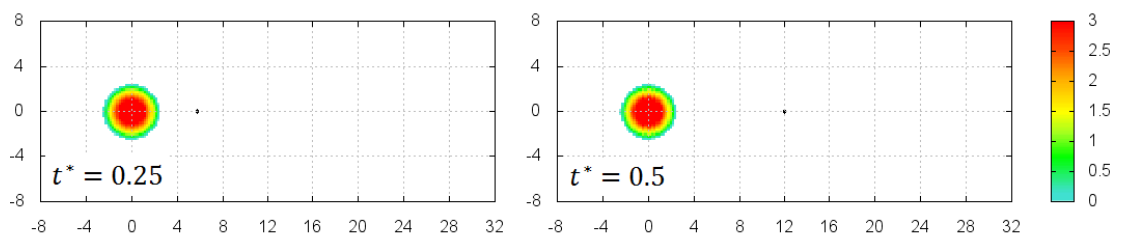

(c) $v_{0}=25$

FIgURE 12. Evolution in time of the biological system for different magnitudes of the directional velocity experienced by the activated/leader cell. (a) $v_{0}=0$, (b) $v_{0}=20$, and (c) $v_{0}=25$. The values of the non-dimensional interaction radii and the non-dimensional interaction forces are given by Eqs. (5.3) and (5.4), respectively.

interactions is not enough to break the inner stability of the continuous aggregate in time to let it follow the leader individual, so that their mutual distance quickly exceeds $\left(R^{12}\right)^{*}=\left(R^{21}\right)^{*}$. It is useful to remark that the setting of the computational algorithm does not impact the simulation outcome: the separation of the leader cell from the inactivated colony is in fact observed also in the case of significantly reduced time steps of the numerical scheme.

In order to obtain a deformation of the continuous colony, it is necessary to modify the interaction force matrix, so that population 2 is not able to reach its inner equilibrium. However, a decrement in $\left(F^{22}\right)^{*}$, the most intuitive option, would correspond to a decrement in the adhesion/repulsion ratio and therefore would only imply a non-realistic explosion of the continuous aggregate, as demonstrated in details in Sec. 3.2. A better way to operate is instead to substantially increase the value of the exogenous interaction strength $\left(F^{21}\right)^{*}$, which, as seen, determines how the dynamics of inactivated cells are influenced by the movement of the differentiated individual. We indeed use the following modified matrix:

$$
\mathbf{F}_{2}^{*}=\left(\begin{array}{cc}
0.1 & 0.1 \\
30 & 0.4
\end{array}\right) \text {. }
$$

With this setting, at large enough values of $v_{0}$ (i.e., $v_{0} \geq 50$ ), it is possible to effectively observe a deformation of the undifferentiated cell population besides the differentiated leader. In particular, as the tip individual moves away, a finger forms and extends from the continuous aggregate, which grows almost at the same velocity as the discrete cell. Eventually, a little continuous mass detaches from the rest of the colony and goes on following the leader, while the remaining part of the finger collapses, see Fig. 13.

However, as we have previously hypothesized, when the preferred velocity drops this threshold of magnitude, the discrete cell is absorbed within the continuous mass, which is again able to reorganize in 

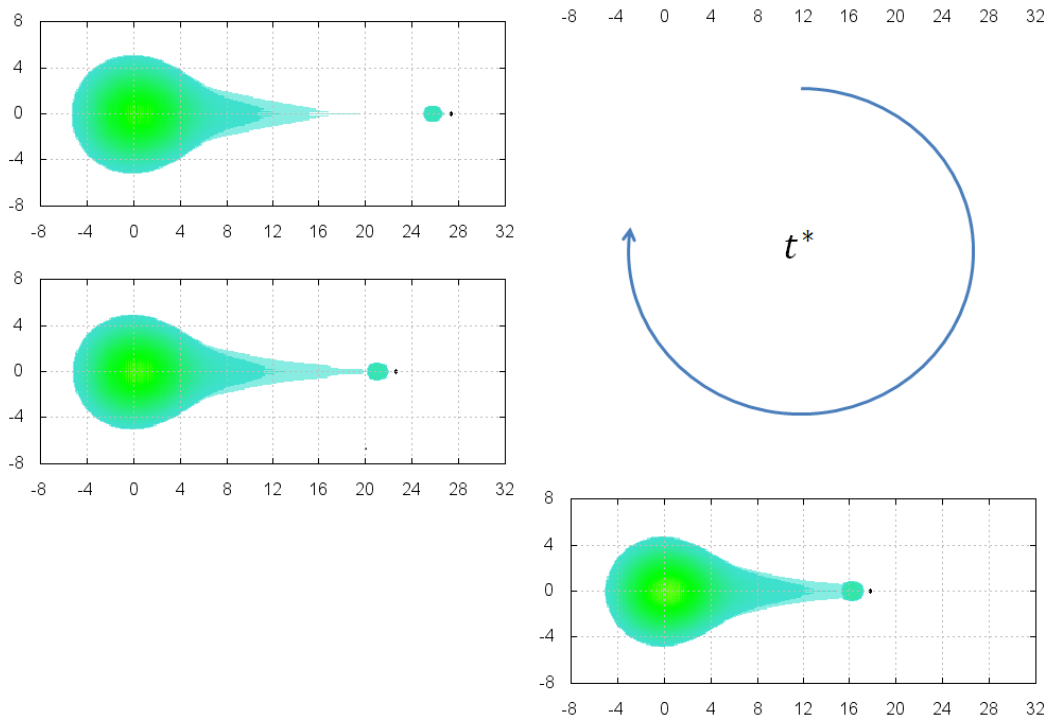
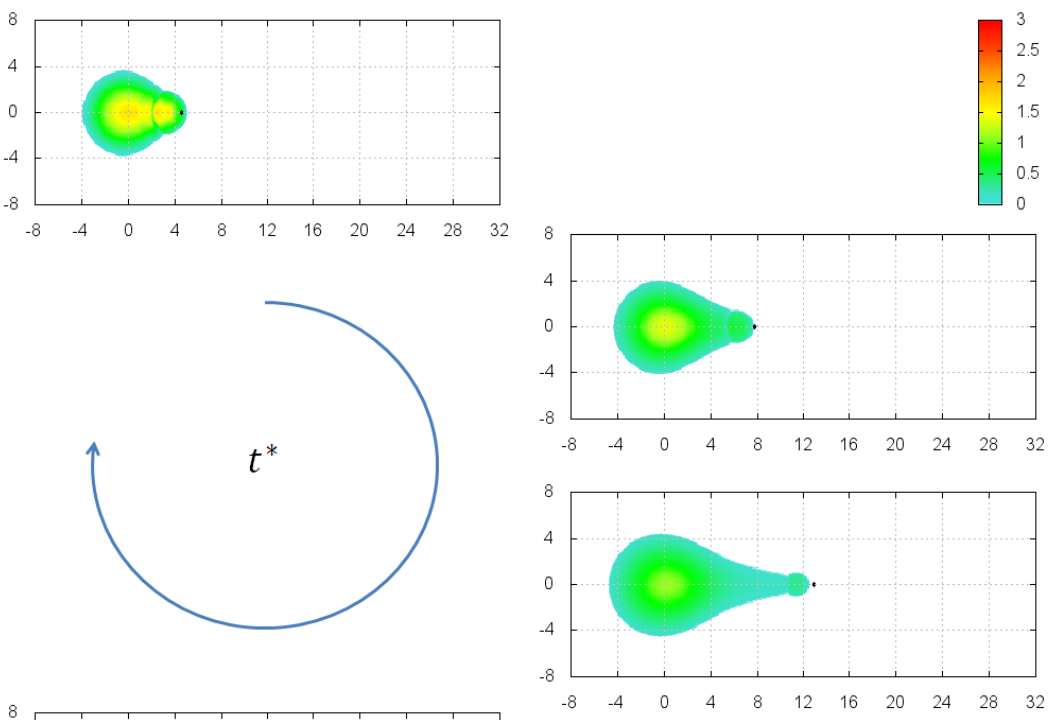

Figure 13. Evolution in time of the biological system in the case of the modified non-dimensional interaction matrix $\mathbf{F}_{2}^{*}$ given in Eq. (5.5). In particular, $v_{0}=50$, while the non-dimensional interaction radii remain as in Eq. (5.3). Clockwise $t^{*}=$ $0.05,0.1,0.2,0.3,0.4,0.5$.

a stable configuration, as reproduced in Fig. 14. In particular, if $v_{0}$ is still sufficiently high (i.e., $>10$ ), the activated cell, regardless of its absorption, is able to move almost the entire continuous colony, that behaves again as a rigid body.

\subsection{Three activated cells with an undifferentiated aggregate}

The same parameter settings introduced in Sec. 5.2 are then tested in the case of three differentiated cells, homogenously distributed around the standard colony of non-activated individuals, see panel (b) of Fig. 11. This biological system is close to reproduce a small aggregate of tumor cells, where microenvironmental substrate gradients, formed within the malignant mass, cause a well-localized phenotypic specification of cancer individuals, which typically differentiate in an outer viable rim of highly metabolic and active cells and an interior core of quiescent individuals. As a trivial extension of the results previously presented, in the absence of any preferred velocity, the non-dimensional interaction matrix $\mathbf{F}_{1}^{*}$ given in (5.4) allows the discrete individuals to remain at the border of the continuous mass, which finds, as usual, its inner stability (not shown). On the opposite, if $\mathbf{F}_{2}^{*}$ is used, the activated cells are absorbed within population 2 (not shown).

Let us now introduce a preferred component in the velocity of the discrete cells, which is radially distributed and depends on their actual position. In particular, the relevance of such a directional velocity is assumed to increase with the distance of the activated individuals from the center of the domain, modeling therefore an increasing abundance of motility factors toward the borders, as, for $i=1,2,3$ :

$$
\mathbf{v}_{d i r}^{* i}\left(\mathbf{x}_{t^{*}, 1}^{* i}\right)=v_{0}\left(\mathbf{x}_{t^{*}, 1}^{* i}-\mathbf{0}\right),
$$

where $\mathbf{0}=(0,0)$. 

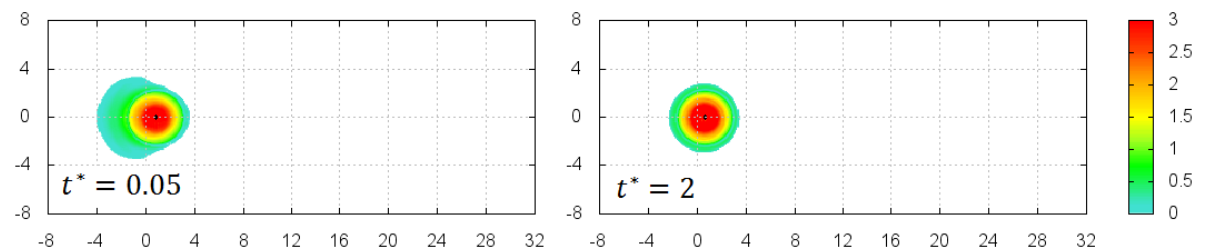

(a) $v_{0}=0$
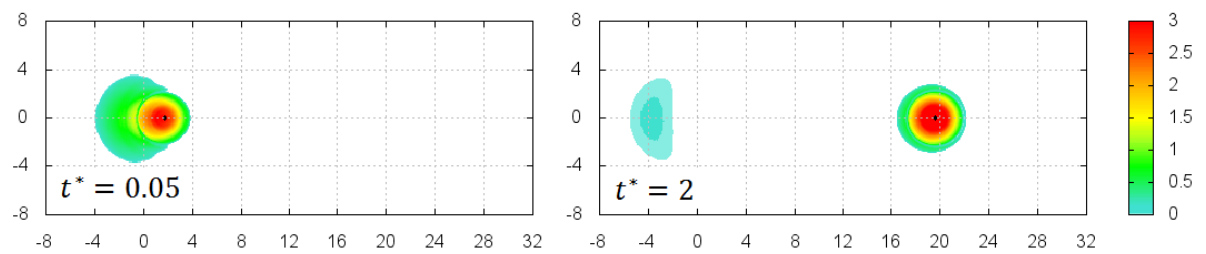

(b) $v_{0}=20$

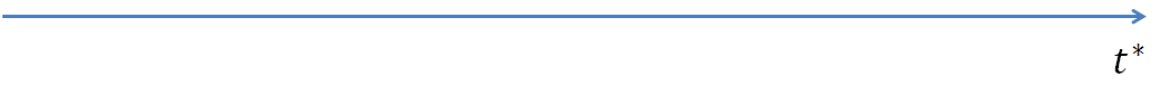

FiguRE 14. Evolution in time of the biological system for different magnitudes of the directional velocity experienced by the activated/leader cell in the case of the modified non-dimensional interaction matrix $\mathbf{F}_{2}^{*}$ given in Eq. (5.5). The non-dimensional interaction radii remain as in Eq. (5.3)

By employing $\mathbf{F}_{1}^{*}$ as the interaction force matrix, we observe that, for low values of $v_{0}$ (say $v_{0} \leq$ 20 ), the discrete individuals rest again around the continuous colony (which only undergoes an inner reorganization, see Fig. 15 (top-left panel)). With respect to the case of $v_{0}=0$, such a behavior is now due to the fact that the undifferentiated aggregate would in principle follow each of the discrete cell (as shown in Fig. 12 (b)): however, the coupling of three attraction forces balance, maintaining the overall system in a sort of equilibrium. At higher values of $v_{0}$, the three differentiated cells escape from the bulk of the continuous spheroid, as in the case of a single activated cell (compare Figs. 15 (bottom-left panel) and $12(\mathrm{c}))$.

If we instead assume $\mathbf{F}_{2}^{*}$ as the intercellular interaction matrix, it is straightforward to notice that, if $v_{0}$ is not sufficiently high (e.g., $<50$ ), the activated cells are retained within the continuous colony and their opposite movements inhibit any expected rigid translation of the system, as reproduced in Fig. 15 (top-right panel). Finally, at $v_{0}=50$, the discrete individuals are able to scatter away from the undifferentiated aggregate followed by three cellular fingers, see Fig. 15 (bottom-right panel). It is useful to remark that the phenomenon of fingering characterizes many malignancies, see for example melanocytic dysplasia in the epidermis overlying the dermal component. In particular, recent studies of photo-micrograph have shown that the "ragged" surfaces of different tumors relate to the overall severity of the disease [18,33]. Tumors with an unstable fingered morphology are in fact typically more aggressive and hard to be treated than smoother ones: even if their invasive depth is limited, they are difficult to be surgically removed.

The phenomenon of fingering has been captured by several modeling approaches. In [40], finger extensions have been reproduced by a cellular automaton model of tumor growth, which takes into account malignant cell motility, proliferation and death, intercellular adhesion, autocrine and paracrine growth stimulation and stromal destruction. In this work, finger fractal dimensionality is related to biophysical determinants of tumor and stromal cells. However, the authors are not able to establish a clear relation between the finger dimensions and evolution and the overall biological behavior of the tumor: some growth properties considered to be associated with an increased degree of disease malignancy influence fractal dimensionality in opposite directions. In [38] and [45], extended versions of the Cellular Potts model reproduce different morphologies of tumor invasion fronts. In both works, malignant cells invade the host 

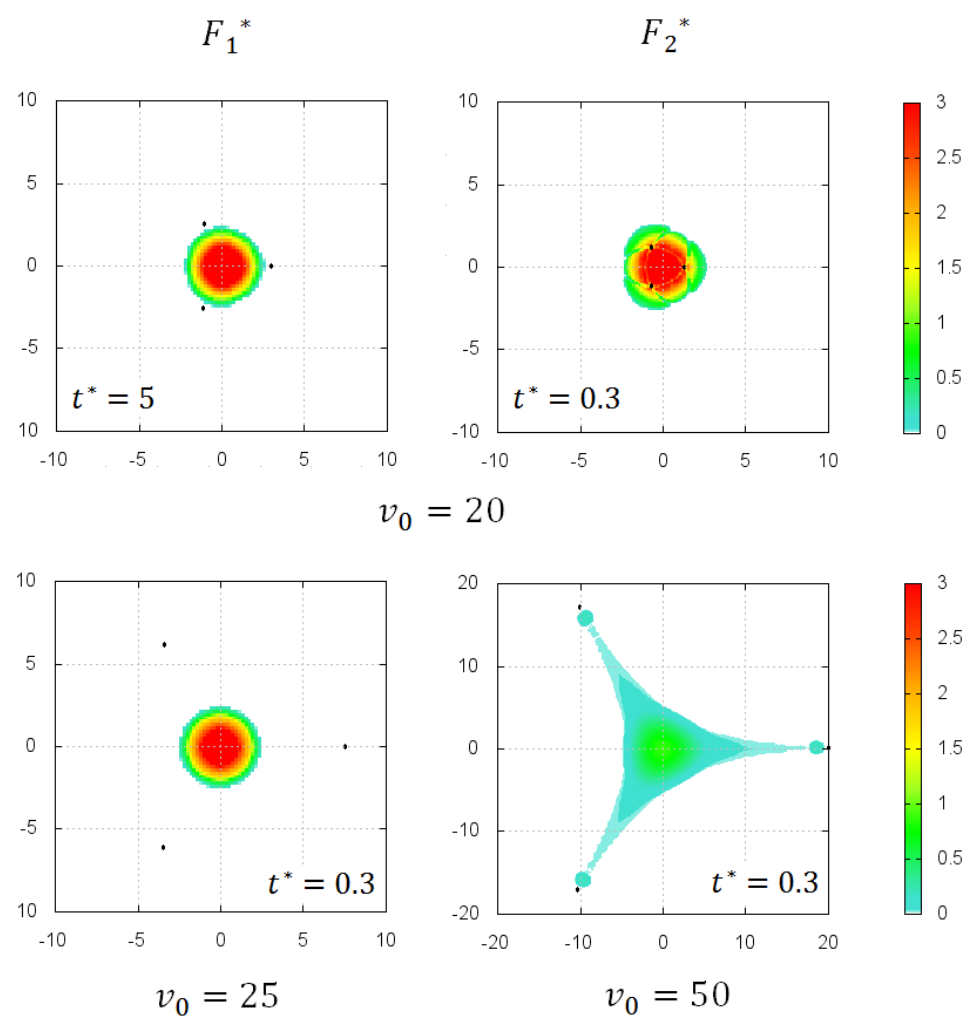

FiguRE 15. Resulting configurations of the biological system, composed of three acrivated/discrete cells and an undifferentiated continuous aggregate, in the case of distinct non-dimensional interaction matrices, as given either by (5.4) or by (5.5). $v_{0}$ measures the intensity of the directional velocity characteristic of the differentiated individuals, while the non-dimensional interaction radii remain as in Eq. (5.3).

tissue via haptotactic mechanisms, that are facilitated by the dissolution of non-soluble matrix proteins. However, in [38] fingering phenomena are observed only when cell proliferation is included, whereas in [45] only when cell division is not taken into account. Entering in more details, in [38], rapid divisions of highly metabolic external individuals results in the formation of little cell islands, which are connected with the main tumor mass by short and thick fingers. On the opposite, in [45], cell proliferation results in a morphological transition of invading tumors, which are no longer characterized by a succession of quite long and thin fingers spreading out into the ECM but rather growing as compact solid masses. In [46], fingering phenomena are instead captured by a diffuse interface continuum model of multispecies tumor growth. The simulations presented therein show that the tumor nonlinearly evolves with a branchedshape configuration. In particular, such a complex structure arises from a diffusional instability, which leads to the creation of buds elongating into protruding fingers. The instability is fundamental for the overall tumor development, as it enables the malignant mass to increase the exposure to nutrient and therefore to overcome the diffusional limitations to growth. With this mechanism, the tumor has in principle the potential to grow indefinitely, as the instability repeats itself on the buds and on the resulting fingers. Finally, in [3], a hybrid discrete-continuum (HDC) model, in which cells are treated as discrete stochastic variables and the microenvironmental parameters as deterministic concentrations, analyzes tumor morphology and phenotypic evolution under different pressure conditions. In particular, invasive masses characterized by fingering margins, which are dominated by few clones with aggressive traits, are observed in harsh conditions of the tumor microenvironment (e.g., hypoxia). It is finally useful to remark that our approach allows instead to reproduce fingering phenomena by only accounting for tip cell directional movement and intercellular adhesion, without any other cellular or molecular processes. 

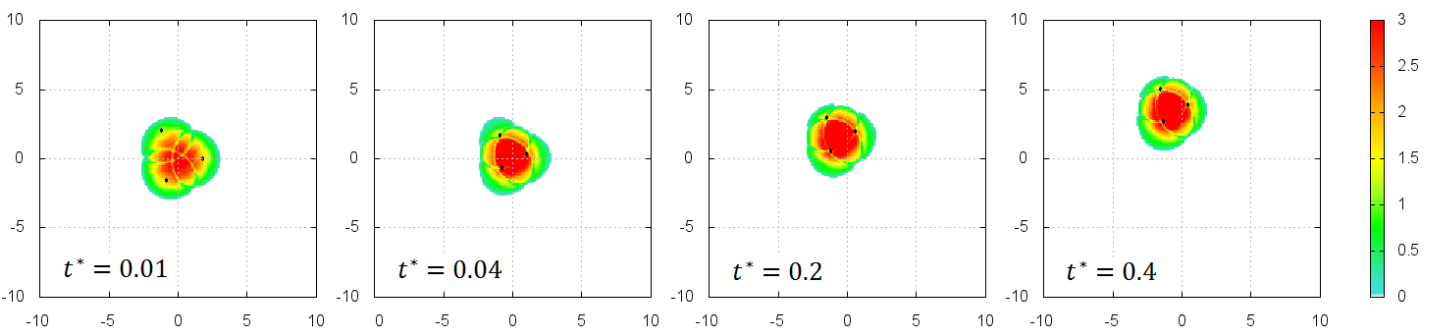

(a) $v_{0}=50$
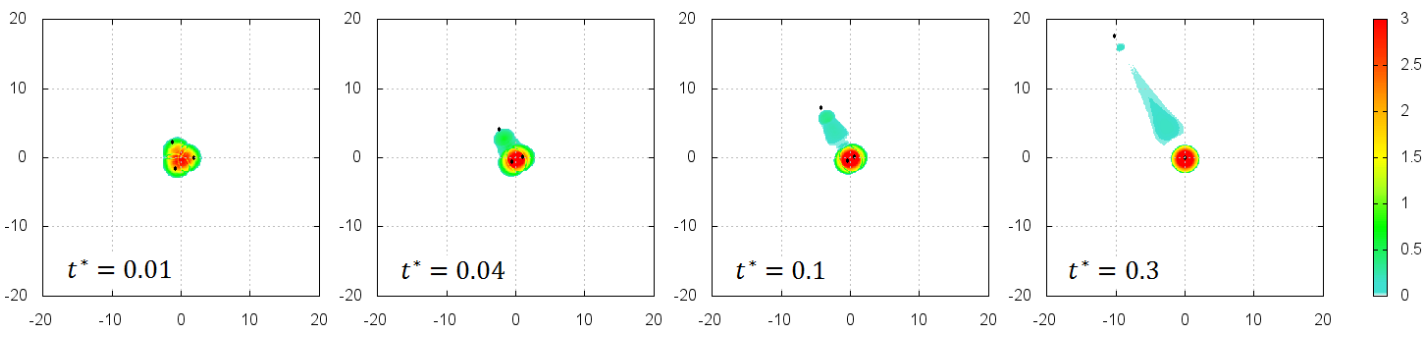

(b) $v_{0}=65$

FIgURE 16. Evolution in time of the biological system for different magnitudes of the directional velocity experienced by only one of the activated cell in the cases of the modified non-dimensional interaction matrix $\mathbf{F}_{2}^{*}$ given in Eq. (5.5). The non-dimensional interaction radii remain as in Eq. (5.3).

In particular, as seen, the fingering dynamics mainly arise from the breakage of the inner equilibrium of the continuous colony due to the exogenous intercellular adhesion. It would be interesting to verify if such a minimal set of mechanisms would be sufficient to reproduce fingering extension also in the above-analyzed computational approaches.

As a final case of interest, we analyze the evolution of the biological system when only one of the three differentiated cells effectively exhibits the radial preferred velocity defined in Eq. (5.6) while the other two stay at rest, i.e., $\mathbf{v}_{d i r}^{*}\left(\mathbf{x}_{t^{*}, 1}^{* i}\right)=\mathbf{0}$ for $i=2,3$. In particular, we use $\mathbf{F}_{2}^{*}$ as the interaction force matrix. Similarly to the previous cases, if $v_{0}$ is not sufficiently high, all the discrete individuals remain absorbed within the non-motile continuous mass (not shown). However, if $v_{0} \approx 50$, the inactivated colony first assumes a stable configuration and then undergoes a quasi-rigid translation dragged by the moving discrete cell, as shown in Fig. 16 (a). Finally, at even higher $v_{0}$, we observe that a small finger elongates and then detaches from the continuous population, following the migrating discrete cell, as shown in Fig. 16 (b). This is due to the fact that the activated individual, moving too fast, quickly overcomes the exogenous interaction distance, while the undifferentiated colony is still reorganizing.

In the previous sets of simulations, the preferred velocity of the differentiated cells is established $a$ priori, being defined by a vector whose direction is constant and whose modulus is either a parameter or a space-dependent variable. Although representing simplified scenarios, the proposed cases have been chosen for their relevant pedagogical usefulness. In fact, they provide a complete screening of model outcomes emerging from different quantitative relationships between the magnitude of $\mathbf{v}_{\text {dir }}$ and of selected exogenous/endogenous intercellular interactions. However, we are aware that more realistic modeling approaches have to determine specific extracellular and intracellular factors that underlie such a preferred velocity. For instance, as commented in Sec. 2.1, cell movement is significantly determined by taxis-like mechanisms. It also relies on intercellular adhesive interactions (in this case, $\mathbf{v}_{\text {dir }}$ should be coupled to a force balance or, at least, related to the intercellular interaction force matrix). Then, there are a wide 
range of biochemical and biophysical determinants specific for differentiated/tip cells that guide collective migration (see [30] for an extensive review). As a hallmark, leading cells are for instance intrinsically polarized, as their front engages with the tissue substrate while their rear region remains engaged with the neighbor cells. Such a polarity is maintained by mechanotransduction through integrins and cytoskeletal elements and is regulated by the activity of intracellular small rho GTPases. This issue may be included in the model by defining for each leader individual a (dynamic) polarity vector, which then influences the relative directional velocity. Tip individuals may also determine their direction of movement through selected adhesive interactions with the matrix environment. After anterior attachment mediated by extending filopodia and/or lamellipodia, which protrude and give rise to focalized adhesions between matrix structures and the intracellular cytoskeleton, pulling forces towards ECM are in fact generated by actomyosin-driven contraction of cell body so that the ECM is displaced and realigned, thereby affecting cell migration. In this case, $\mathbf{v}_{\text {dir }}$ may indeed be referred to adhesive and/or mechanical properties of the substrate. Finally, leader cells execute pericellular proteolysis to create or enlarge physical paths for migration. This is particularly relevant in the case of 3D matrices characterized by abundant and/or rigid fibers [29]. It is however straightforward to remark that more realistic descriptions of tip cell migration can be achieved by an explicit modeling of selected biochemical, biophysical and/or structural properties of the extracellular environment. This may represent a very interesting improvement of the proposed approach, in particular, from an applied-oriented viewpoint.

\section{Discussion}

All biological phenomena emerge from the intricate interaction between different levels of organization. For instance, the macroscopic evolution of cell aggregates is determined by the behavior and the biophysical properties of single cells, as well as by their mutual interactions. On the opposite, specific dynamics occurring at the supercellular level feed back to the microscopic phenomenology of single individuals.

A fundamental feature of mathematical approaches for biological/biomedical problems is indeed the ability to describe and reproduce such a multiscale framework. According to this idea, we have here introduced a mathematical method, based on measure theory, intended to provide an Eulerian ensemble representation of a cell colony starting from a Lagrangian individual characterization of a microscopic test entity. More technically, the approach consists in modeling cell positions in the physical space as random variables and then in looking at the evolution of their probability distribution rather than at that of the random variables themselves. This way, the underlying physical system is always regarded as a microscopic granular one but it is possible to obtain its Eulerian representation (i.e., as a whole) in term of an evolving measure. In other words, the key idea of the method consists in a change of viewpoint: from the physical positions of the cells to the probability measure attached to them. It is worth noticing that, once the latter is available, all important information on the system, such as mass distribution and spatial patterns, is directly recoverable without going back to the detail of the aforesaid random variables. Therefore, the latter mainly serves as a conceptual tool for deducing coherently a transport equation of the probability distribution. A subsequent specification of the global mass measure (i.e., atomic, absolutely continuous with respect to Lebesgue, or hybrid) then allow to switch between different types/levels of description of the system.

In our opinion, this method offers several conceptual advantages over classical models. First, it enables one to unambiguously transfer microscopic concepts, such as cell interaction radii or chemical sensitivity, into a macroscopic description of the cell distribution.

Secondly, the use of measure theory allows to make definitely the difference with respect to more traditional approaches, where the "discrete vs. continuous" dichotomy is referred to the spatial scale at which the system is modelled (i.e., "microscopic vs. macroscopic"). The discrete and continuous representations stemming from such a formalism are in fact two copies of the same mathematical model, featuring only two different levels of spatial detail. It is also possible to suitably couple them, thereby giving rise to a blended discrete-continuous model for the spatio-temporal evolution of the distribution of cells, in which the discrete granularity and the continuous flow complement each other toward a multiscale description of cell dynamics. Our approach is also suitable to address, both analytically and computationally, large cell aggregates while retaining the microscopic characterization of the single components. All these features allows to distinguish the proposed mathematical framework and classical approaches presented in the literature, which typically rely on the idea that the continuous description 
emerges in the limit from a vanishing discrete description, and is thus a good approximation of the latter for very large numbers of dimensionless particles. This is the case of mathematical frameworks employing mean-field limits [11] and/or heuristic laws of large numbers [8] to pass from microscopic models of individual dynamics to macroscopic models for the evolution of the corresponding system as a whole. In literature, also coarse-graining procedures are used to switch from cell automaton models to the corresponding continuous approximation (i.e., via multivariate master equations) [21]. Rescaling techniques are the bases for i) parameter calibration of agent-based models (ABMs) from experimental data at the cell-scale (immunohistochemistry of pathology specimens) and ii) for deriving coarse-grained proliferation and apoptotic rates at the macroscopic tissue scale from the microscopic level of ABMs themselves [34]. Finally, macroscopic mathematical formulations of nonspatial and spatially-distributed dynamics of selected lattice-gas cellular automaton (LGCA) are derived in [5] to study the role of the migration/proliferation dichotomy in glioma invasion at the avascular stage. Interestingly, the authors exhaustively comment the first formulation corresponds to a well-stirred system while the second one is based on the Chapman-Enskog expansion method and yields to a reaction-diffusion equations (RDE) system.

Thanks to the proper level of mathematical abstraction provided by measure theory, we also expect to obtain dynamical predictions which, whereas not being completely biased toward specific realizations as purely individual-based models would, include however a right amount of empirical characterization of the system (for instance, the intrinsic granularity of a cell aggregate) within the theoretical level of the mathematical description (for instance, the time evolution of probability density functions). In this respect, after a renormalization with respect to the total number of the cells $N$, the measure $\mu_{t}$ can be understood as the probability measure linked to the distribution of individuals. In this way, the proposed approach can be interpreted as a probabilistic framework, which is conceptually suited to deal with a statistical characterization of the evolution of biological systems, constituting a significant advancement with respect to both purely deterministic average macroscopic models and stochastic eventbased microscopic models. In more details, most mathematical models currently available in literature adopt a deterministic point of view: once initial and/or boundary conditions are given, the evolution of the system is univocally determined by the model. Possible randomness in the prescribed data is not explicitly taken into account, and consequently it is not dynamically transferred to the model's predictions, unless several repeated simulations and a posteriori averages are performed, starting each time from different randomly generated conditions. However, such a technique is generally not efficient, as it is affected by inevitable fluctuations caused both by sampling of the input data and by averaging of the output results over partly arbitrary space and/or time domains. Such a criticism applies both to macroscopic models, which, by definition, look at average deterministic quantities, but also to microscopic models. In fact, although they may complement deterministic dynamics with some random noise, the latter allows only for stochasticity on single realizations rather than for outcomes in terms of probability distributions whence to extract at once statistics of practical interest. This is a crucial drawback, since biological systems are characterized by an objective unpredictability of several background conditions, which impose an evaluation in terms of probability evolution of the system of interest.

The illustrative simulations presented in the paper have then shown nontrivial consequences of the multiscale dynamics on the predicted pattern formation, thus implying that the scale used to represent the underlying physical system is a crucial aspect in theoretical biology. In particular, we have provided the fact that, in a specific range of parameter values (i.e., high intercellular interaction forces and low intercellular distances), discrete, continuous and hybrid descriptions of the system give the same dynamics. On the opposite, outside this set of values, each type of mathematical representation determines a distinct evolution of the simulated system. In particular, as commented along the text, the discrete approach is closer to real phenomenologies.

The proposed theoretical approach is also particularly suited to deal with problems in which the interplay between different scales of mathematical description can be linked to the coexistence of multiple cell populations, or of multiple differentiated clones of the same cell line. In these cases, it is in fact necessary to treat each set of individuals at a distinct scale according to its intrinsic properties. To this regard, as a relevant sample application of such a model extension, we have studied a biological system composed of an inactivated/undifferentiated cell colony, properly described with a continuous approximation, and of a small number of activated/differentiated cells, singularly reproduced with a 
discrete viewpoint. In particular, the resulting realizations have shown that the evolution of the overall system is mainly driven by the behavior of the activated cells, which guide the inactivated population. In more details, the emerging patterns arise from the strength of the exogenous interactions, as its value determines whether the activated individuals are free to scatter in the neighboring space (eventually followed by fingers of the undifferentiated population) or are forced to be absorbed by the continuous mass itself. The hybrid coupling of continuum-discrete descriptions, here implemented in the two-population cell system, is a very promising modeling approach for biological/biomedical problems. However, most of existing modeling frameworks typically use a discrete representation for cell-scale elements and continuum approximations to describe the evolution of sub-cellular molecular variables (e.g., nutrients, oxygen and diffusible factors, see $[7,37])$. However, there is an increasing number of works that differ from this line of research, i.e., that relate different types of mathematical representation to phenotypically different cell subpopulations. For example, in [31], the authors present a hybrid model for the study of the evolution of the ductal carcinoma in situ (DCIS, an early non-invasive stage of breast cancer), in which epithelial cells (ECs) are modeled individually while the ECM forming the tumor microenvironment (TME) is treated as a continuum element. Some hybrid models, developed to study the evolution of tumor spheroids, employ instead different descriptions of malignant cells in different regions of the tumor mass. For instance, in $[32,42]$, an agent-based model (ABM) is implemented to describe the proliferating and highly metabolic malignant cells at the spheroid edge (i.e., their interactions, morphology and internal dynamics), while a continuum viscoelastic model is used for the quiescent and necrotic regions within the bulk of the tumor mass. Analogously, in [43], an ABM describes as a discrete sizeless points invading tumor cells of glioma tumors, whereas the rest of the malignant mass is approached with a continuum model suitable to maintain a spherical symmetry. Moreover, in [23], an interesting hybrid mathematical environment studies the hypoxia-induced epithelial-to-mesenchymal transition during tumor growth. An ABM is in fact used to represented differentiated mesenchymal cells and coupled to a continuum model for undifferentiated epithelial volume fractions. In particular, discrete cells are set to be produced in hypoxic regions and can be locally converted back to the continuum description if their amount exceeds a given threshold. However, in all these cases, the coupling among the discrete and continuous components is limited to the production/reabsorption of discrete cells into undifferentiated continuous regions of tumors through mass and momentum fluxes. In fact, explicit dynamical influences between discrete and continuous elements are not included. Moreover, differently from our measure-based approach, the dynamics of continuous components are not upscaled and derived from realistic cell-scale behavior. Finally, in [12], the authors propose a modeling approach for tumor growth based on Dynamic Density Functional Theory (DDFT), which is able to provide a mesoscale continuum framework directly derived from a stochastic discrete model. Such a mathematical framework is extended in order to account different cell subpopulations, characterized by different phenotypes and birth/death processes. The authors also discuss how the proposed continuum and discrete systems can coexist and integrate to obtain hybrid models. It is useful to remark that the DDFT-based approach is able to account for correlations at the continuum scale: this make its difference with respect to standard mean-field models cited in the previous part of this discussion, which usually employ uncorrelated mean-field approximations.

For all the above-cited reasons, we expect our method to give an interesting contribution in the development of multiscale approaches to the modeling of cell phenomena. However, the presented mathematical framework has still some important limitations, whose improvements would represent relevant modeling refinements. First, a wide range of parameter settings results in non-realistic model outcomes, although consistent through the different types of descriptions (cf. Fig. 10). This issue may be addressed either by implementing more appropriate interaction kernels or by deriving empirical relations between selected model parameters and experimentally measurable quantities. As mentioned in the text, it would be also interesting to describe in more details cell interactions with the extracellular environment, for example with the substrate and/or with diffusive molecular variables. In this respect, more sophisticated boundary conditions may be needed in the case of applications dealing with more realistic domains (i.e., analysis of blood flows, collective cell migration in three-dimensional matrix scaffolds, organogenesis). Then, in the present form of the approach, cell duplication/death, as well as cell differentiation in the case of multiple coexisting populations, are not included. This of course represents a relevant issue for a detailed simulation of a wide spectrum of biological phenomena (for example, morphogenesis, tumor growth, wound healing). However, taking into account such processes would require significant revisions 
of the analytic architecture underlying the model: the resulting mass measures in fact would no longer evolve satisfying conservation equations, as localized source/sink terms should be included. In particular, the passage from the phenomenological laws of test individuals to the Eulerian models of the dynamics of cell populations should no longer imply only the use of conservation and transport theorems.

A further interesting model perspective regards the role of the multiscale coupling parameter $u$. In the present form of the model, $u$ is in fact assumed to be constant in time and homogeneous in space, thereby establishing a priori the contribution of the microscopic and the macroscopic scale to the evolution of the system. However, in theoretical biology, it is often more appropriate to use different mathematical descriptions in different specific subdomains and/or to dynamically change the scale of representation of the system. For example, inactivated cells located in a given part of the domain may activate in response to external or internal stimuli, thereby requiring a local switch from a continuous description to a discrete representation. This amounts to convert $u$ into a function of $\mathbf{x}$ and possibly also of $t$, while maintaining its value in the interval $[0,1]$. In this way, the evolution of $u_{t}(\mathbf{x})$ may be determined by the evolution of selected microenvironmental variables, such as ions, proteins, growth factors, which for instance suggest the actual local scale of description of the system (i.e., $u_{t}(\mathbf{x})=u_{t}\left(c_{t}(\mathbf{x})\right.$ ), where $c$ is the local amount of the molecular variable of interest). It is also possible to argue that an appropriate tuning of $u$ may be inherited from information on the local cell density, as $u_{t}(\mathbf{x})=u_{t}\left(N_{t}(\mathbf{x})\right)$, where $N_{t}(\mathbf{x})$ identifies the actual number of individuals located in $\mathbf{x}$. This may implement the assumption that decrements in the number of cells correspond to increment in the effect of their localized individualities. Non-constant values of $u$, besides proper constitutive laws determining its spatiotemporal evolution, would also require non-trivial technicalities to derive the resulting multiscale system (2.20). In fact, it would be necessary to deal also with the time and space derivaties of $u$. In particular, this would ultimately cause the hybrid transport velocity to be no longer a formal linear combination of the velocities obtained for the discrete and the continuous descriptive instances of the system.

Acknowledgements. MS was funded by a post-doctoral research scholarship awarded by the National Institute for Advanced Mathematics "F. Severi" (INdAM, Italy).

\section{References}

[1] B. Alberts, A. Johnson, J. Lewis, M. Raff, K. Roberts, P. Walter. Molecular Biology of the Cell, 4th ed. Garland Science, New York, 2002.

[2] A. R. A. Anderson, M. A. J. Chaplain, K. A. Rejniak, Eds. Single-cell-based models in biology and medicine. Birkäuser, Boston, 2007.

[3] A. R. A. Anderson, A. M. Weaver, P. T. Cummings, V. Quaranta. Tumor morphology and phenotypic evolution driven by selective pressure from the microenvironment. Cell, 127 (2006), 905-915.

[4] N. Bellomo, B. Piccoli, A. Tosin. Modeling crowd dynamics from a complex system viewpoint. Math. Methods Appl. Sci., 22 (2012), 1230004.

[5] K. Böttger, H. Hatzikirou, A. Chauviere, A. Deutsch. Investigation of the migration/proliferation dichotomy and its impact on avascular glioma invasion. Math. Model. Nat. Phenom., 7 (2012), 105-135.

[6] F. Bussolino, M. Arese, E. Audero, E. Giraudo, S. Marchio, S. Mitola, L. Primo, G. Serini, 2003. Biological aspects in tumor angiogenesis. In Preziosi, L., Ed., Cancer modeling and simulation, Mathematical Biology and Medicine Sciences, Chapman and Hall/CRC, 1-16.

[7] V. Capasso and D. Morale. Stochastic modelling of tumour-induced angiogenesis. J. Math. Biol., 58 (2009), $219-233$.

[8] V. Capasso and D. Morale. Asymptotic behavior of a system of stochastic particles subject to nonlocal interactions, Stoch. Anal. Appl., 27 (2009), 574-603.

[9] P. Carmeliet, R. K. Jain. Angiogenesis in cancer and other diseases. Nature, 407 (2000), 249-257.

[10] P. Carmeliet. Angiogenesis in life, disease and medicine. Nature, 438 (2005), 932-936.

[11] J. A. Carrillo, M. Fornasier, G. Toscani, F. Vecil (2010) Particle, kinetic, and hydrodynamic models of swarming, in Naldi, G., Pareschi, L. and Toscani, G., Eds., Mathematical Modeling of Collective Behavior in Socio-Economic and Life Sciences, Modeling and Simulation in Science, Engineering and Technology, Birkäuser, 297-336.

[12] A. Chauviere, H. Hatzikirou, I. G. Kevrekidis, J. S. Lowengrub, V. Cristini. Dynamic density functional theory of solid tumor growth: preliminary models. AIP Advances, 2 (2012), 011210.

[13] A. Colombi, M. Scianna, A. Tosin. Differentiated cell behavior: A multiscale approach using measure theory. Submitted for publication. Preprint at arXiv:1108.1212.

[14] E. Cristiani, B. Piccoli, A. Tosin. Multiscale modeling of granular flows with application to crowd dynamics. Multiscale Model. Simul., 9 (2011), 155-182.

[15] E. Cristiani, P. Frasca, B. Piccoli. Effects of anisotropic interactions on the structure of animal groups. J. Math. Biol., 62 (2011), 569-588. 
[16] E. Cristiani, B. Piccoli, A. Tosin. Modeling self-organization in pedestrians and animal groups from macroscopic and microscopic viewpoints. In G. Naldi, L. Pareschi, and G. Toscani, Eds., Mathematical Modeling of Collective Behavior in Socio-Economic and Life Sciences. Modeling and Simulation in Science, Engineering and Technology, Birkhäuser, Boston, 337-364, 2010.

[17] V. Cristini, J. Lowengrub, Eds. Multiscale modeling of cancer: an integrated experimental and mathematical modeling approach. Cambridge University Press, 2011.

[18] S. S. Cross. Fractals in pathology. J. Pathol., 182 (1997), 1-8.

[19] A. Deutsch, S. Dormann. Cellular automaton modeling of biological pattern formation: characterization, applications, and analysis. Birkäuser, Boston, 2005.

[20] D. Drasdo. On selected individual-based approaches to the dynamics of multicellular systems. In W. Alt and M. Griebel, Eds., Multiscale Modeling. Birkhäuser, Boston, 169-203, 2005.

[21] D. Drasdo. Coarse graining in simulated cell populations. Adv. Complex Syst., 8 (2005), 319-363.

[22] D. Drasdo, S. Hohme. Individual-based approaches to birth and death in avascular tumors. Math. Comp. Model, 37 (2003), 1163-1175.

[23] H. B. Frieboes, F. Jin, Y. L. Chuang, S. M. Wise, J. S. Lowengrub, V. Cristini. Three-dimensional multispecies nonlinear tumor growth-II: Tumor invasion and angiogenesis. J. Theor. Biol., 264 (2010), 1254-1278.

[24] P. Friedl, P. B. Noble, P. A. Walton, D. W. Laird, P. J. Chauvin, R. J. Tabah, M. Black, K. S. Zänker. Migration of coordinated cell clusters in mesenchymal and epithelial cancer explants in vitro. Cancer Res., 55 (1995), 4557-4560.

[25] P. Friedl, D. Gilmour. Collective cell migration in morphogenesis, regeneration and cancer. Nat. Rev. Mol. Cell. Biol., 10 (2009), 445-457.

[26] F. Graner, J. A. Glazier. Simulation of biological cell sorting using a two dimensional extended Potts model. Phys. Rev. Lett., 69 (1992), 2013-2017.

[27] Y. Hegerfeldt, M. Tusch, E. B. Bröcker, P. Friedl. Collective cell movement in primary melanoma explants: plasticity of cell-cell interaction, $\beta 1$-integrin function, and migration strategies. Cancer Res., 62 (2002), 2125-2130.

[28] O. Ilina, P. Friedl. Mechanisms of collective cell migration at a glance. J. Cell Sci., 122 (2009), 3203-3208.

[29] O. Ilina, G.-J. Bakker, A. Vasaturo, R. M. Hoffman, P. Friedl. Two-photon laser-generated microtracks in 3D collagen lattices: principles of MMP-dependent and -independent collective cancer cell invasion. Phys. Biol., 8 (2011), 015010.

[30] A. A. Khalila, P. Friedl. Determinants of leader cells in collective cell migration. Integr. Biol., 2 (2010), 568-574.

[31] Y. Kim, H. G. Othmer. A hybrid model of tumor-stromal interactions in breast cancer. Bull. Math. Biol. 75 (2013), 1304-1350.

[32] Y. Kim, M. A. Stolarska, H. G. Othmer. A hybrid model for tumor spheroid growth in vitro i: theoretical development and early results. Math. Models Methods Appl. Sci. 17 (2007), 1773.

[33] G. Landini, Y. Hirayama, T. J. Li, M. Kitano. Increased fractal complexity of the epithelial-connective tissue interface in the tongue of 4 NQO-treated rats. Pathol. Res. Pract., 196 (2000), 251-258.

[34] P. Macklin, M. E. Edgerton, A. M. Thompson, V. Cristini. Patient-calibrated agent-based modelling of ductal carcinoma in situ (DCIS): From microscopic measurements to macroscopic predictions of clinical progression. J. Theor. Biol., 301 (2012), 122-140.

[35] B. Piccoli, A. Tosin. Time-evolving measures and macroscopic modeling of pedestrian flow. Arch. Ration. Mech. Anal., 199 (2011), 707-738.

[36] L. Preziosi, A. Tosin. Multiphase and multiscale trends in cancer modelling. Math. Model. Nat. Phenom., 4 (2009), $1-11$.

[37] M. Scianna, L. Preziosi. Multiscale Developments of the Cellular Potts Model. Multiscale Model. Simul., 10 (2012), $342-382$.

[38] M. Scianna, L. Preziosi. A hybrid Cellular Potts Model describing different morphologies of tumor invasion fronts. Math. Model. Nat. Phenom., 7 (2012), 78-104.

[39] M. Scianna, L. Preziosi. Cellular Potts Models: Multiscale Developments and Biological Applications. Chapman and Hall/CRC Press (2013)

[40] J. Smolle. Fractal tumor stromal border in a nonequilibrium growth model. Anal. Quant. Cytol. Histol., 20 (1998), $7-13$.

[41] W. G. Stetler-Stevenson, S. Aznavoorian, L. A. Liotta, Tumor cell interactions with the extracellular matrix during invasion and metastasis. Ann. Rev. Cell Biol. 9 (1993), 541-573.

[42] M. A. Stolarska, Y. Kim, H. G. Othmer. Multi-scale models of cell and tissue dynamics. Phil. Trans. R. Soc. A, 367 (2009), 3525-3553.

[43] M. L. Tanaka, W. Debinski, I. K. Puri. Hybrid mathematical model of glioma progression. Cell Prolif., 42 (2009), 637-646.

[44] A. Tosin, P. Frasca, Existence and approximation of probability measure solutions to models of collective behaviors. Netw. Heterog. Media, 6 (2011), 561-596.

[45] S. Turner, J. A. Sherratt. Intercellular adhesion and cancer invasion: A discrete simulation using the extended Potts model. J. Theor. Biol., 216 (2002), 85-100.

[46] S. M. Wise, J. S. Lowengrub, H. B. Frieboes, V. Cristini. Three-dimensional multispecies nonlinear tumor growth-I Model and numerical method. J. Theor. Biol., 253 (2008), 524-543.

[47] J. M. Zahm, H. Kaplan, A. Herard, F. Doriot, D. Pierrot, P. Somelette, E. Puchelle Cell migration and proliferation during the in vitro repair of the respiratory epithelium. Cell. Motil. Cytoskel., 37 (1997), 33-43.

[48] Y. W. Zhang, G. F. Vande Woude. HGF/SF-Met signaling in the control of branching morphogenesis and invasion. J. Cell. Biochem., 88 (2003), 408-417. 